The Journal of Fourier Analysis and Applications

Volume 12, Issue 3, 2006

\title{
Density, Overcompleteness, and Localization of Frames. II. Gabor Systems
}

\author{
Radu Balan, Peter G. Casazza, Christopher Heil, and Zeph Landau \\ Communicated by Karlheinz Gröchenig
}

\begin{abstract}
This work develops a quantitative framework for describing the overcompleteness of a large class of frames. A previous article introduced notions of localization and approximation between two frames $\mathcal{F}=\left\{f_{i}\right\}_{i \in I}$ and $\mathcal{E}=\left\{e_{j}\right\}_{j \in G}$ ( $G$ a discrete abelian group), relating the decay of the expansion of the elements of $\mathcal{F}$ in terms of the elements of $\mathcal{E}$ via a map a: $I \rightarrow G$. This article shows that those abstract results yield an array of new implications for irregular Gabor frames. Additionally, various Nyquist density results for Gabor frames are recovered as special cases, and in the process both their meaning and implications are clarified. New results are obtained on the excess and overcompleteness of Gabor frames, on the relationship between frame bounds and density, and on the structure of the dual frame of an irregular Gabor frame. More generally, these results apply both to Gabor frames and to systems of Gabor molecules, whose elements share only a common envelope of concentration in the time-frequency plane.

The notions of localization and related approximation properties are a spectrum of ideas that quantify the degree to which elements of one frame can be approximated by elements of another frame. In this article, a comprehensive examination of the interrelations among these localization and approximation concepts is made, with most implications shown to be sharp.
\end{abstract}

\section{Introduction}

The fundamental structural feature of a frame that is not a Riesz basis is the overcompleteness of its elements. To date, even partial understanding of this overcompleteness has been restricted to limited examples, such as finite-dimensional frames or highly structured

Math Subject Classifications. 42C15, 46C99.

Keywords and Phrases. Density, excess, frames, Gabor systems, modulation spaces, overcompleteness, Riesz bases, wavelets, Weyl-Heisenberg systems.

Acknowledgments and Notes. The second author was partially supported by NSF Grants DMS0102686 and DMS-0405376. The third author was partially supported by NSF Grant DMS-0139261. The fourth author was partially supported by The City University of New York PSC-CUNY Research Award Program.

(C) 2006 Birkhäuser Boston. All rights reserved ISSN 1069-5869 DOI: $10.1007 /$ s00041-005-5035-4 
("lattice") frames of windowed exponentials or of time-frequency shifts (Gabor systems). Together, the ideas and results presented in this article and in [4] provide a quantitative framework for describing the overcompleteness of a large class of frames. The consequences of these ideas are: (a) an array of fundamental new results for frames that hold in a general setting, (b) significant new results for the case of Gabor frames, as well as a new framing of existing results that clarifies their meaning, and (c) the presentation of a novel and fruitful point of view for future research.

Our approach begins with two frames $\mathcal{F}=\left\{f_{i}\right\}_{i \in I}$ and $\mathcal{E}=\left\{e_{j}\right\}_{j \in G}$, where $G$ is a discrete abelian group; we then introduce a notion of the localization of $\mathcal{F}$ with respect to $\mathcal{E}$. The idea of localization is that it describes the decay of the coefficients of the expansion of elements of $\mathcal{F}$ in terms of the elements of $\mathcal{E}$. To make this notion of decay meaningful, a map $a$ from the index set $I$ into the index set $G$ is introduced. With this setup, we establish a remarkable equality between three seemingly unrelated quantities: Certain averages of $\left\langle f_{i}, \tilde{f}_{i}\right\rangle$ and $\left\langle e_{j}, \tilde{e}_{j}\right\rangle$ of frame elements with corresponding canonical dual frame elements, which we refer to as relative measures, and the density of the set $a(I)$ in $G$ [4, Theorem 5]. This equality between density and relative measure is striking since the relative measure is a function of the frame elements, while the density is solely determined by the index set $I$ and the mapping $a: I \rightarrow G$.

Due to the length of this work, it is natural to present it in two parts. The first part, containing the theoretical and structural results that have driven the research, appeared in [4] (hereafter referred to as "Part I"). In this article (the second part) we accomplish the following two main goals.

(1) We apply the theoretical results to the case of Gabor systems

$$
\mathcal{G}(g, \Lambda)=\left\{M_{\omega} T_{x} g\right\}_{(x, \omega) \in \Lambda}=\left\{e^{2 \pi i \omega \cdot t} g(t-x)\right\}_{(x, \omega) \in \Lambda},
$$

which yields a collection of new results that can be summarized as follows.

(a) Functions with time-frequency concentration generate localized Gabor frames (Theorem 2). We show how the degree of localization of a Gabor frame is tied to the time-frequency concentration of the generating window function or "atom" $g$. This alone yields a significant improvement over what was previously known about the approximation properties of irregular Gabor frames. We extend these results to more general systems of Gabor molecules whose elements are not required to be simple time-frequency shifts of each other, but instead need only share a common envelope of concentration about points in the time-frequency plane.

(b) Structure of the dual frame of a Gabor frame (Theorem 7). We prove that if an irregular Gabor frame is generated by a function $g$ which is sufficiently concentrated in the time-frequency plane (specifically, $g$ lies in the modulation space $M^{1}$ ), then the elements of the dual frame also lie in $M^{1}$. We further prove that the dual frame forms a set of Gabor molecules, and thus, while it need not form a Gabor frame, the elements do share a common envelope of concentration in the time-frequency plane. Moreover, this same result applies if the original frame was only itself a frame of Gabor molecules.

(c) A relationship between density of time-frequency shifts and inner products of frame elements (Theorems 3,5). We apply the core abstract result of Part I, the DensityRelative Measure Theorem [4, Theorem 5]. This implies a remarkable equality between seemingly unrelated quantities: The density of the time-frequency shifts of a Gabor frame and certain averages of inner products between Gabor frame 
elements and the canonical dual frame elements. As a consequence we obtain new relationships between the density of the index set, the frame bounds, and the norm of the window of the Gabor frame.

(d) The excess of Gabor frames (Theorem 6). We show that in any overcomplete Gabor frame or set of Gabor molecules, a set of frame elements with positive density can be removed yet still leave a frame.

(2) We provide a comprehensive examination of the interrelations among the suite of localization and approximation concepts introduced in Part I, and in most cases provided counterexamples showing that these implications are sharp.

We believe that localization is a powerful and useful new concept. As evidence of this fact, we note that Gröchenig has independently introduced a concept of localized frames, for a completely different purpose, in [37]. We learned of Gröchenig's results shortly after completion of our own major results. The definitions of localizations presented here and in [37] differ, but the fact that this single concept has independently arisen for two very distinct applications shows its utility. In his elegant article, Gröchenig has shown that frames which are sufficiently localized in his sense provide frame expansions not only for the Hilbert space $H$ but for an entire family of associated Banach function spaces. Gröchenig further showed that if a frame is sufficiently localized in his sense (a polynomial or exponential localization) then the dual frame is similarly localized.

\subsection{Outline}

We briefly review some results known for irregular Gabor frames that are related to the themes of this article and then discuss our new contributions.

There is an extensive literature available for "lattice" Gabor systems of the form $\mathcal{G}\left(g, \alpha \mathbf{Z}^{d} \times \beta \mathbf{Z}^{d}\right)$ or $\mathcal{G}\left(g, A\left(\mathbf{Z}^{2 d}\right)\right.$ ), where $A$ is an invertible $2 d \times 2 d$ matrix (even so, many basic questions remain open even lattice Gabor frames). In contrast, until quite recently very few results were available for irregular Gabor systems $\mathcal{G}(g, \Lambda)$ where $\Lambda$ is an arbitrary subset of $\mathbf{R}^{2 d}$. An early article by Gröchenig [34] provided some partial sufficient conditions for the existence of irregular Gabor frames. The articles [52, 55, 14] proved a Nyquist Density Theorem for irregular Gabor frames, which can be stated as follows:

(a) If $\mathcal{G}(g, \Lambda)$ is a frame for $L^{2}\left(\mathbf{R}^{d}\right)$, then $1 \leq D_{B}^{-}(\Lambda) \leq D_{B}^{+}(\Lambda)<\infty$;

(b) If $\mathcal{G}(g, \Lambda)$ is a Riesz basis for $L^{2}\left(\mathbf{R}^{d}\right)$, then $D_{B}^{-}(\Lambda)=D_{B}^{+}(\Lambda)=1$.

Here $D_{B}^{ \pm}(\Lambda)$ are the lower and upper Beurling densities of $\Lambda$, which are defined precisely in Example 2. H. Landau [52] proved statement (a) with extra hypotheses assumed on $g$. Ramanathan and Steger [55] proved statements (a) and (b) with extra hypotheses assumed on $\Lambda$. A complete proof was given in [14] (inspired by the results in [41]). Additional density-related results for irregular Gabor frames were given by Janssen in [49]. Some recent articles on irregular Gabor frames are [20, 15, 42, 60, 3, 53, 61, 37].

In the case that $\Lambda$ is a rectangular lattice of the form $\Lambda=\alpha \mathbf{Z}^{d} \times \beta \mathbf{Z}^{d}$, several alternative proofs and extensions of the Density Theorem are known, see [56, 1, 16, 47, 19, 8]. For additional history and references see the exposition in $[17,7,35,44]$. Note that a lattice $\Lambda=\alpha \mathbf{Z}^{d} \times \beta \mathbf{Z}^{d}$ has uniform Beurling densities $D_{B}^{+}\left(\alpha \mathbf{Z}^{d} \times \beta \mathbf{Z}^{d}\right)=D_{B}^{-}\left(\alpha \mathbf{Z}^{d} \times \beta \mathbf{Z}^{d}\right)=$ $1 /(\alpha \beta)^{d}$.

Ramanathan and Steger's approach to the Density Theorem showed that it is not the algebraic structure of the lattice as such that is essential, but rather the fact that Gabor 
frames satisfy a certain Homogeneous Approximation Property (essentially the weak HAP presented in this article, using as a reference system a Gabor frame with a Gaussian generating function). For a Gabor frame, the HAP essentially states that the rate of approximation of a given function by Gabor frame elements is invariant under time-frequency shifts of the function. This is remarkable in the case of irregular Gabor frames, since there is no structure to relate the specific frame elements used in the approximation of one time-frequency shift $M_{q} T_{p} f$ to those in an approximation of another time-frequency shift of $f$. The HAP is a fundamental property of Gabor frames, yet very few articles subsequent to [55] have made use of it.

We investigate the localization properties of irregular Gabor frames in Section 3. Following the introduction of some notation in Section 3.1, we show in Section 3.2 that every Gabor system has at least a weak amount of localization ( $\ell^{2}$-row decay) with respect to the Gabor orthonormal basis generated by the box function, and as a consequence, we recover the fact that every Gabor Bessel sequence has finite density.

In Section 3.3 we show that much stronger localization is obtained if the reference Gabor system is generated by a function that is well-concentrated in the time-frequency plane. The degree of concentration is quantified by the modulation spaces, which are the natural function spaces associated with Gabor analysis. In particular, we show that if $\mathcal{G}(g, \Lambda)$ is an arbitrary Gabor system such that $g$ lies in the modulation space $M^{p}$ $(1 \leq p \leq 2)$, and $\mathcal{G}\left(\phi, \alpha \mathbf{Z}^{d} \times \beta \mathbf{Z}^{d}\right)$ is a reference Gabor system whose generator $\phi$ lies in the modulation space $M^{1}$, then $\left(\mathcal{G}(g, \Lambda), a, \mathcal{G}\left(\phi, \alpha \mathbf{Z}^{d} \times \beta \mathbf{Z}^{d}\right)\right)$ is $\ell^{p}$-localized. Additionally, $(\mathcal{G}(g, \Lambda), a)$ is $\ell^{1}$-self-localized if $p=1$. In particular, by the implications established in Theorem 10, $\ell^{2}$-localization for a frame implies both the Strong HAP and the Strong Dual HAP, so these results greatly improve the Homogeneous Approximation Property previously established for Gabor frames in [55, 14].

In Section 4 we use this extended knowledge of the localization properties of Gabor systems to derive new implications for irregular Gabor frames. In Section 4.1 we recover the density results for Gabor frames given above, and furthermore we extend the meaning of density by showing that the Beurling densities of $\Lambda$ are related to the measures of the Gabor frame. We show that the upper and lower measures of a Gabor frame satisfy $\mathcal{M}^{ \pm}(\mathcal{G}(g, \Lambda))=$ $1 / D_{B}^{\mp}(\Lambda)$. This gives a new interpretation of the density of a Gabor frame, and as a consequence we obtain new relations among the density of the index set, the frame bounds, and the norm of the generator. In particular, we show that if $\mathcal{G}(g, \Lambda)$ is a tight frame then the index set $\Lambda$ must have uniform Beurling density, i.e., $D_{B}^{-}(\Lambda)=D_{B}^{+}(\Lambda)$. Thus tight Gabor frames require a certain amount of uniformity in the index set.

Until [2], it was not known whether every overcomplete lattice Gabor frame $\mathcal{G}\left(g, \alpha \mathbf{Z}^{d} \times\right.$ $\beta \mathbf{Z}^{d}$ ) that was not a Riesz basis contained an infinite subset that could be removed yet leave a frame. In Section 4.2, we show that if $\mathcal{G}(g, \Lambda)$ is a Gabor frame whose generator $g$ lies in $M^{1}$, then whenever $D_{B}^{-}(\Lambda)>1$, there is not merely an infinite subset but a subset with positive density which may be removed from the frame yet still leave a frame.

In Section 4.3 we address the fundamental question of the structure of the canonical dual frame of an irregular Gabor frame. As is well-known, the canonical dual frame of a lattice Gabor frame is again a lattice Gabor frame, indexed by the same lattice. This need not be the case for an irregular Gabor frame $\mathcal{G}(g, \Lambda)=\left\{e^{2 \pi i \omega \cdot t} g(t-x)\right\}_{(x, \omega) \in \Lambda}$ with an arbitrary index set $\Lambda$. A canonical dual frame $\tilde{\mathcal{G}}=\left\{\tilde{g}_{x, \omega}\right\}_{(x, \omega) \in \Lambda}$ will exist, but to date, except for results in [37], essentially nothing has been known about this dual beyond the fact that each dual frame element $\tilde{g}_{x, \omega}$ is some function in $L^{2}\left(\mathbf{R}^{d}\right)$. We prove that if $g$ possesses sufficient time-frequency concentration, namely that $g \in M^{1}$, then each $\tilde{g}_{x, \omega}$ possesses the same concentration, i.e., $\tilde{g}_{x, \omega} \in M^{1}$. For the case of lattice systems $\left(\Lambda=\alpha \mathbf{Z}^{d} \times \beta \mathbf{Z}^{d}\right)$, 
this result was previously obtained by Gröchenig and Leinert [40]. However, in addition to extending to the completely irregular setting, we also prove that the dual frame $\tilde{\mathcal{G}}$ is Gabor-like. Namely, even though $\tilde{\mathcal{G}}$ need not itself be a Gabor frame, we show that it will form a set of Gabor molecules, meaning that each function $\tilde{g}_{x, \omega}$ is concentrated in the time-frequency plane about the point $(x, \omega)$ with a common envelope of concentration for each $(x, \omega) \in \Lambda$. In fact, this result holds even if the original frame was only itself a frame of Gabor molecules.

We conclude the Gabor portion of this article in Section 4.4 where we observe that most of the results obtained for Gabor frames carry over with minor changes to the case of Gabor molecules.

For simplicity of presentation, most of our results will be stated for the case of Gabor frames for all of $L^{2}\left(\mathbf{R}^{d}\right)$, but many of them can be extended to the case of Gabor frame sequences, or to Gabor frames with multiple generators, by making use of the machinery developed in Part I and this article. Results analogous to the ones formulated for Gabor systems could also be formulated for the case of windowed exponentials.

Finally, in Section 5 we carefully examine the interrelations between the range of localization properties and approximation concepts that have been used to develop the theory. A set of approximation properties for abstract frames introduced in Part I is given in Definition 4. These are defined in terms of how well the elements of the reference system are approximated by finite linear combinations of frame elements, or vice versa, and provide an abstraction for general frames of the essential features of the Homogeneous Approximation Property (HAP) that is known to hold for Gabor frames or windowed exponentials (see $[55,41,14])$. Theorem 10 establishes an extensive list of implications that hold among the localization and approximation properties, and additionally in most cases we provide examples which show that these implications are sharp. In particular, there is an equivalence between $\ell^{2}$-column decay and the HAP, and between $\ell^{2}$-row decay and a dual HAP.

\subsection{General Notation}

We use the notation from Part I, which we briefly review here. $H$ will refer to a separable Hilbert space. The frame or system of interest will be indexed by a countable index set $I$. The reference frame or system will be indexed by an additive discrete group $G$ of the form

$$
G=\prod_{i=1}^{d} a_{i} \mathbf{Z} \times \prod_{j=1}^{e} \mathbf{z}_{n_{j}} .
$$

We define a metric on $G$ as follows. If $m_{j} \in \mathbf{Z}_{n_{j}}$, set $\delta\left(m_{j}\right)=0$ if $m_{j}=0$, otherwise $\delta\left(m_{j}\right)=1$. Then given $g=\left(a_{1} n_{1}, \ldots, a_{d} n_{d}, m_{1}, \ldots, m_{e}\right) \in G$, set

$$
|g|=\sup \left\{\left|a_{1} n_{1}\right|, \ldots,\left|a_{d} n_{d}\right|, \delta\left(m_{1}\right), \ldots, \delta\left(m_{e}\right)\right\} .
$$

The metric is $d(g, h)=|g-h|$ for $g, h \in G$. The reader can simply take $G=\mathbf{Z}^{d}$ without much loss of insight on a first reading (the metric in this case is simply the $\ell^{\infty}$ metric on $\mathbf{R}^{d}$ restricted to $\mathbf{Z}^{d}$ ).

We implicitly assume that there exists a map $a: I \rightarrow G$ associated with $I$ and $G$. This map will often not be injective. For each integer $N>0$ we let

$$
S_{N}(j)=\left\{k \in G:|k-j| \leq \frac{N}{2}\right\}
$$


denote a discrete "cube" or "box" in $G$ centered at $j \in G$. The cardinality of $S_{N}(j)$ is independent of $j$. For example, if $G=\mathbf{Z}^{d}$ then $\left|S_{2 N}(j)\right|=\left|S_{2 N+1}(j)\right|=(2 N+1)^{d}$. We let $I_{N}(j)$ denote the inverse image of $S_{N}(j)$ under $a$, i.e.,

$$
I_{N}(j)=a^{-1}\left(S_{N}(j)\right)=\left\{i \in I: a(i) \in S_{N}(j)\right\} .
$$

\subsection{Notation for Frames and Riesz Bases}

We use standard notations for frames and Riesz bases as found in the texts $[13,17,35,62]$ or the research-tutorials $[11,45]$.

A sequence $\mathcal{F}=\left\{f_{i}\right\}_{i \in I}$ is a frame for $H$ if there exist frame bounds $A, B>0$ such that $A\|f\|^{2} \leq \sum_{i \in I}\left|\left\langle f, f_{i}\right\rangle\right|^{2} \leq B\|f\|^{2}$ for all $f \in H$. The analysis operator $T: H \rightarrow \ell^{2}(I)$ is $T f=\left\{\left\langle f, f_{i}\right\rangle\right\}_{i \in I}$, and its adjoint $T^{*} c=\sum_{i \in I} c_{i} f_{i}$ is the synthesis operator. The Gram matrix is $T T^{*}=\left[\left\langle f_{i}, f_{j}\right\rangle\right]_{i, j \in I}$. The frame operator $S f=T^{*} T f=\sum_{i \in I}\left\langle f, f_{i}\right\rangle f_{i}$ is a bounded, positive, and invertible mapping of $H$ onto itself. The canonical dual frame is $\tilde{\mathcal{F}}=S^{-1}(\mathcal{F})=\left\{\tilde{f}_{i}\right\}_{i \in I}$ where $\tilde{f}_{i}=S^{-1} f_{i}$. We call $\mathcal{F}$ a tight frame if we can take $A=B$, and a Parseval frame if we can take $A=B=1$. If $\mathcal{F}$ is any frame, then $S^{-1 / 2}(\mathcal{F})$ is the canonical Parseval frame associated to $\mathcal{F}$. We call $\mathcal{F}$ a uniform norm frame if all the frame elements have identical norms, i.e., if $\left\|f_{i}\right\|=$ const. for all $i \in I$. A frame is norm-bounded below if $\inf _{i}\left\|f_{i}\right\|>0$.

A sequence which satisfies the upper frame bound estimate, but not necessarily the lower estimate, is called a Bessel sequence and B is a Bessel bound.

A sequence $\mathcal{F}=\left\{f_{i}\right\}_{i \in I}$ that is a frame for its closed linear span in $H$ is called a frame sequence. In this case $\tilde{\mathcal{F}}=\left\{\tilde{f}_{i}\right\}_{i \in I}$ will denote its canonical dual frame within $\overline{\operatorname{span}}(F)$, and $P_{\mathcal{F}}$ will denote the orthogonal projection of $H$ onto $\overline{\operatorname{span}}(\mathcal{F})$.

A frame is a basis if and only if it is a Riesz basis. A Riesz sequence is a sequence that forms a Riesz basis for its closed linear span in $H$.

\section{Density, Localization, HAP, and Relative Measure}

In this section we recall basic definitions from Part I, and show how they are implemented for the case of Gabor systems. In Theorem 10 we will derive an extended set of implications that hold among these properties, and provide examples in Section 5.2 showing that most of those implications are sharp.

\subsection{Density}

Given an index set $I$ and a map $a: I \rightarrow G$, we define the density of $I$ by computing the Beurling density of its image $a(I)$ as a subset of $G$. Note that we regard $I$ as a sequence, and hence repetitions of images count in determining the density.

Definition 1 (Density). The lower and upper densities of I with respect to a are

$$
D^{-}(I, a)=\liminf _{N \rightarrow \infty} \inf _{j \in G} \frac{\left|I_{N}(j)\right|}{\left|S_{N}(j)\right|}
$$

and

$$
D^{+}(I, a)=\limsup _{N \rightarrow \infty} \sup _{j \in G} \frac{\left|I_{N}(j)\right|}{\left|S_{N}(j)\right|}
$$


respectively. These quantities could be zero or infinite, i.e., $0 \leq D^{-}(I, a) \leq D^{+}(I, a) \leq \infty$. When $D^{-}(I, a)=D^{+}(I, a)=D$ we say $I$ has uniform density $D$.

These lower and upper densities are only the extremes of the possible densities that we could naturally assign to $I$ with respect to $a$. In particular, instead of taking the infimum or supremum over all possible centers in (2.1) and (2.2) we could choose one specific sequence of centers, and instead of computing the liminf or limsup we could consider the limit with respect to some ultrafilter (some background on ultrafilters is provided in Appendix A). The different possible choices of ultrafilters and sequences of centers gives us the following natural collection of definitions of density.

Definition 2. Let $p$ be a free ultrafilter, and let $c=\left(c_{N}\right)_{N \in \mathbf{N}}$ be any sequence of centers $c_{N} \in G$. Then the density of $I$ with respect to $a, p$, and $c$ is

$$
D(p, c)=D(p, c ; I, a)=p \text {-lim } \frac{\left|I_{N}\left(c_{N}\right)\right|}{\left|S_{N}\left(c_{N}\right)\right|} .
$$

Example 1. If $I=G$ and $a$ is the identity map, then $I_{N}(j)=S_{N}(j)$ for every $N$ and $j$, and hence $D(p, c)=D^{-}(I, a)=D^{+}(I, a)=1$ for every choice of free ultrafilter $p$ and sequence of centers $c$.

It follows from basic properties of ultrafilters that we always have $D^{-}(I, a) \leq D(p, c) \leq$ $D^{+}(I, a)$. It is shown in $\left[4\right.$, Lemma 1] that there exist free ultrafilters $p^{-}, p^{+}$and sequence of centers $c^{-}=\left(c_{N}^{-}\right)_{N \in \mathbf{N}}, c^{+}=\left(c_{N}^{+}\right)_{N \in \mathbf{N}}$ in $G$ such that $D^{-}(I, a)=D\left(p^{-}, c^{-}\right)$and $D^{+}(I, a)=D\left(p^{+}, c^{+}\right)$.

Example 2 (Gabor Systems). Consider an arbitrary Gabor system $\mathcal{F}=\mathcal{G}(g, \Lambda)$ and a reference lattice Gabor system $\mathcal{E}=\mathcal{G}\left(\phi, \alpha \mathbf{Z}^{d} \times \beta \mathbf{Z}^{d}\right)$. The index set $I=\Lambda$ is a countable sequence of points in $\mathbf{R}^{2 d}$, and the reference group is $G=\alpha \mathbf{Z}^{d} \times \beta \mathbf{Z}^{d}$. A natural map $a: \Lambda \rightarrow G$, that we will employ whenever dealing with Gabor systems, is rounding to a near element of $G$, i.e.,

$$
a(x, \omega)=\left(\alpha \operatorname{Int}\left(\frac{x}{\alpha}\right), \beta \operatorname{Int}\left(\frac{\omega}{\beta}\right)\right), \quad(x, \omega) \in \Lambda,
$$

where $\operatorname{Int}(x)=\left(\left\lfloor x_{1}\right\rfloor, \ldots,\left\lfloor x_{d}\right\rfloor\right)$. With this setup, $S_{N}(j)$ is the intersection of $\alpha \mathbf{Z}^{d} \times \beta \mathbf{Z}^{d}$ with the cube $Q_{N}(j)$ in $\mathbf{R}^{2 d}$ centered at $j$ with side lengths $N$. Such a cube contains approximately $N^{2 d} /(\alpha \beta)^{d}$ points of $\alpha \mathbf{Z}^{d} \times \beta \mathbf{Z}^{d}$; precisely,

$$
\lim _{N \rightarrow \infty} \frac{\left|S_{N}(j)\right|}{N^{2 d}}=\frac{1}{(\alpha \beta)^{d}} .
$$

Likewise, because $a$ is a bounded perturbation of the identity map, the number of points in $I_{N}(j)$ is asymptotically the cardinality of $\Lambda \cap Q_{N}(j)$. Consequently, the standard definition of the upper Beurling density $D_{B}^{+}(\Lambda)$ of $\Lambda$ is related to our definition of the upper density of $\Lambda$ with respect to $a$ as follows:

$$
\begin{aligned}
D_{B}^{+}(\Lambda) & =\limsup _{N \rightarrow \infty} \sup _{j \in \mathbf{R}^{2 d}} \frac{\left|\Lambda \cap Q_{N}(j)\right|}{N^{2 d}} \\
& =\frac{1}{(\alpha \beta)^{d}} \limsup _{N \rightarrow \infty} \sup _{j \in \alpha \mathbf{Z}^{d} \times \beta \mathbf{Z}^{d}} \frac{\left|I_{N}(j)\right|}{\left|S_{N}(j)\right|}=\frac{1}{(\alpha \beta)^{d}} D^{+}(\Lambda, a) .
\end{aligned}
$$


Similarly the lower Beurling density of $\Lambda$ is $D_{B}^{-}(\Lambda)=(\alpha \beta)^{-d} D^{-}(\Lambda, a)$. In particular, when $\alpha \beta=1$ (the "critical density" case), our definition coincides with Beurling density, but in general the extra factor of $(\alpha \beta)^{d}$ must be taken into account.

The map $a$ given in (2.3) is the one we will use when dealing with Gabor systems, but any bounded perturbation of $a$ would serve just as well. That is, given $\delta>0$, we could map $(x, \omega)$ to any point in $G=\alpha \mathbf{Z}^{d} \times \beta \mathbf{Z}^{d}$ that is within a distance $\delta$ of $\left(\alpha \operatorname{Int}\left(\frac{x}{\alpha}\right), \beta \operatorname{Int}\left(\frac{\omega}{\beta}\right)\right)$ without any change in the results.

\subsection{The Localization Properties}

The words "column" and "row" in the following definition refer to the $I \times G$ cross-Grammian matrix $\left[\left\langle f_{i}, e_{j}\right\rangle\right]_{i \in I, j \in G}$. We think of the elements in locations $(i, a(i))$ as corresponding to the main diagonal of this matrix.

Definition 3 (Localization). Let $\mathcal{F}=\left\{f_{i}\right\}_{i \in I}$ and $\mathcal{E}=\left\{e_{j}\right\}_{j \in G}$ be sequences in $H$ and $a: I \rightarrow G$ an associated map.

(a) We say $\mathcal{F}$ is $\ell^{p}$-localized with respect to the reference sequence $\mathcal{E}$ and the map $a$, or simply that $(\mathcal{F}, a, \mathcal{E})$ is $\ell^{p}$-localized, if

$$
\sum_{j \in G} \sup _{i \in I}\left|\left\langle f_{i}, e_{j+a(i)}\right\rangle\right|^{p}<\infty .
$$

Equivalently, there must exist an $r \in \ell^{p}(G)$ such that

$$
\forall i \in I, \quad \forall j \in G, \quad\left|\left\langle f_{i}, e_{j}\right\rangle\right| \leq r_{a(i)-j} .
$$

(b) We say that $(\mathcal{F}, a, \mathcal{E})$ has $\ell^{p}$-column decay if for every $\varepsilon>0$ there is an integer $N_{\varepsilon}>0$ so that

$$
\forall j \in G, \quad \sum_{i \in I \backslash I_{N_{\varepsilon}}(j)}\left|\left\langle f_{i}, e_{j}\right\rangle\right|^{p}<\varepsilon .
$$

(c) We say $(\mathcal{F}, a, \mathcal{E})$ has $\ell^{p}$-row decay if for every $\varepsilon>0$ there is an integer $N_{\varepsilon}>0$ so that

$$
\forall i \in I, \quad \sum_{j \in G \backslash S_{N_{\varepsilon}}(a(i))}\left|\left\langle f_{i}, e_{j}\right\rangle\right|^{p}<\varepsilon .
$$

Note that given a sequence $\mathcal{F}$, the definition of localization is dependent upon both the choice of reference sequence $\mathcal{E}$ and the map $a$.

Example 3 (Gabor Systems). For motivation, consider the especially simple case of Gabor systems both indexed by $\mathbf{Z}^{2 d}$, i.e., $\mathcal{F}=\mathcal{G}\left(g, \mathbf{Z}^{2 d}\right)=\left\{M_{n} T_{k} g\right\}_{(k, n) \in \mathbf{Z}^{2 d}}$ and $\mathcal{E}=$ $\mathcal{G}\left(\phi, \mathbf{Z}^{2 d}\right)=\left\{M_{n} T_{k} \phi\right\}_{(k, n) \in \mathbf{Z}^{2 d}}$. In this case, the map $a: \mathbf{Z}^{2 d} \rightarrow \mathbf{Z}^{2 d}$ given by (2.3) is the identity map, and the $\mathbf{Z}^{2 d} \times \mathbf{Z}^{2 d}$ cross-Grammian matrix

$$
\left[\left\langle M_{m} T_{j} g, M_{n} T_{k} \phi\right\rangle\right]_{(j, m) \in \mathbf{Z}^{2 d},(k, n) \in \mathbf{Z}^{2 d}}=\left[\left\langle g, M_{n-m} T_{k-j} \phi\right\rangle\right]_{(j, m) \in \mathbf{Z}^{2 d},(k, n) \in \mathbf{Z}^{2 d}}
$$

is Toeplitz. Set $r_{k, n}=\left|\left\langle g, M_{n} T_{k} \phi\right\rangle\right|$. If $\mathcal{E}=\mathcal{G}\left(\phi, \mathbf{Z}^{2 d}\right)$ is a Bessel sequence with Bessel bound $B$, then

$$
\sum_{(k, n) \in \mathbf{Z}^{2 d}} r_{k, n}^{2}=\sum_{(k, n) \in \mathbf{Z}^{2 d}}\left|\left\langle g, M_{n} T_{k} \phi\right\rangle\right|^{2} \leq B\|g\|_{2}^{2}<\infty,
$$


so $r \in \ell^{2}\left(\mathbf{Z}^{2 d}\right)$, and hence $\left(\mathcal{G}\left(g, \mathbf{Z}^{2 d}\right), a, \mathcal{G}\left(\phi, \mathbf{Z}^{2 d}\right)\right)$ is $\ell^{2}$-localized.

Unfortunately, Gabor frames indexed by $\alpha \mathbf{Z}^{d} \times \beta \mathbf{Z}^{d}$ with $\alpha \beta=1$ are not very useful in practice. It can easily be shown via Zak transform techniques that if such a system is a frame for $L^{2}\left(\mathbf{R}^{d}\right)$ then it will be a Riesz basis. However, the Balian-Low Theorem (BLT) states that the generator $g$ of such a Gabor Riesz basis cannot be simultaneously well-concentrated in both time and frequency. For exposition and references on the BLT see the survey [7]. Some recent results on the BLT in higher dimensions are in $[6,39]$.

Therefore, consider an arbitrary Gabor system

$$
\mathcal{F}=\mathcal{G}(g, \Lambda)=\left\{M_{\omega} T_{x} g\right\}_{(x, \omega) \in \Lambda},
$$

where $\Lambda \subset \mathbf{R}^{2 d}$. For a reference system take a lattice Gabor system of the form $\mathcal{E}=$ $\mathcal{G}\left(\phi, \alpha \mathbf{Z}^{d} \times \beta \mathbf{Z}^{d}\right)=\left\{M_{\eta} T_{u} \phi\right\}_{(u, \eta) \in \alpha \mathbf{Z}^{d} \times \beta \mathbf{Z}^{d}}$, where $\alpha, \beta>0$. We regard $\mathcal{E}$ as being indexed by $G=\alpha \mathbf{Z}^{d} \times \beta \mathbf{Z}^{d}$ and use the natural map $a: \Lambda \rightarrow G$ given in (2.3) that sends an element of $\Lambda$ to a near element of $G$. It is no longer the case that the cross-Grammian matrix $\left[\left\langle M_{\omega} T_{x} g, M_{\eta} T_{u} \phi\right\rangle\right]_{(x, \omega) \in \Lambda,(u, \eta) \in G}$ is Toeplitz, but we will show in Theorem 2 that if $\Lambda$ has finite density and $\phi$ possesses a certain amount of joint concentration in time and frequency then $\left(\mathcal{G}(g, \Lambda), a, \mathcal{G}\left(\phi, \alpha \mathbf{Z}^{d} \times \beta \mathbf{Z}^{d}\right)\right)$ is $\ell^{2}$-localized. The specific requirement on $\phi$ is that it must lie in the modulation space $M^{1}\left(\mathbf{R}^{d}\right)$, which is defined precisely in Section 3.3. Moreover, the localization can be improved by also imposing a time-frequency concentration condition on $g$. Specifically, we show in Theorem 2 that if $g \in M^{p}\left(\mathbf{R}^{d}\right)$ and $\phi \in M^{1}\left(\mathbf{R}^{d}\right)$, then $\left(\mathcal{G}(g, \Lambda), a, \mathcal{G}\left(\phi, \alpha \mathbf{Z}^{d} \times \beta \mathbf{Z}^{d}\right)\right)$ is $\ell^{p}$-localized.

Remark 1. For comparison, let us give Gröchenig's notion of localization from [37]. Let $I$ and $J$ be countable index sets in $\mathbf{R}^{d}$ that are separated, i.e., $\inf _{i \neq j \in I}|i-j|>0$ and similarly for $J$. Then $\mathcal{F}=\left\{f_{i}\right\}_{i \in I}$ is s-polynomially localized with respect to a Riesz basis $\mathcal{E}=\left\{e_{j}\right\}_{j \in J}$ if for every $i \in I$ and $j \in J$ we have

$$
\left|\left\langle f_{i}, e_{j}\right\rangle\right| \leq C(1+|i-j|)^{-s} \quad \text { and } \quad\left|\left\langle f_{i}, \tilde{e}_{j}\right\rangle\right| \leq C(1+|i-j|)^{-s},
$$

where $\left\{\tilde{e}_{j}\right\}_{j \in J}$ is the dual basis to $\left\{e_{j}\right\}_{j \in J}$. Likewise $\mathcal{F}=\left\{f_{i}\right\}_{i \in I}$ is exponentially localized with respect to a Riesz basis $\mathcal{E}=\left\{e_{j}\right\}_{j \in J}$ if for some $\alpha>0$ we have for every $i \in I$ and $j \in J$ that

$$
\left|\left\langle f_{i}, e_{j}\right\rangle\right| \leq C e^{-\alpha|i-j|} \quad \text { and } \quad\left|\left\langle f_{i}, \tilde{e}_{j}\right\rangle\right| \leq C e^{-\alpha|i-j|} .
$$

\subsection{The Approximation Properties}

The following approximation properties extract the essence of the Homogeneous Approximation Property that is satisfied by Gabor frames, but without reference to the exact structure of Gabor frames.

Definition 4 (Homogeneous Approximation Properties). Let $\mathcal{F}=\left\{f_{i}\right\}_{i \in I}$ be a frame for $H$ with canonical dual $\tilde{\mathcal{F}}=\left\{\tilde{f}_{i}\right\}_{i \in I}$, and let $\mathcal{E}=\left\{e_{j}\right\}_{j \in G}$ be a sequence in $H$. Let $a: I \rightarrow G$ be an associated map.

(a) We say $(\mathcal{F}, a, \mathcal{E})$ has the weak $H A P$ if for every $\varepsilon>0$, there is an integer $N_{\varepsilon}>0$ so that for every $j \in G$ we have $\operatorname{dist}\left(e_{j}, \overline{\operatorname{span}}\left\{\tilde{f}_{i}: i \in I_{N_{\varepsilon}}(j)\right\}\right)<\varepsilon$. Equivalently, there must exist scalars $c_{i, j}$, with only finitely many nonzero, such that

$$
\left\|e_{j}-\sum_{i \in I_{N_{\varepsilon}}(j)} c_{i, j} \tilde{f}_{i}\right\|<\varepsilon
$$


(b) We say $(\mathcal{F}, a, \mathcal{E})$ has the strong $H A P$ if for every $\varepsilon>0$, there is an integer $N_{\varepsilon}>0$ so that for every $j \in G$ we have

$$
\left\|e_{j}-\sum_{i \in I_{N_{\varepsilon}}(j)}\left\langle e_{j}, f_{i}\right\rangle \tilde{f}_{i}\right\|<\varepsilon .
$$

Definition 5 (Dual Homogeneous Approximation Properties). Let $\mathcal{F}=\left\{f_{i}\right\}_{i \in I}$ be a sequence in $H$, and let $\mathcal{E}=\left\{e_{j}\right\}_{j \in G}$ be a frame for $H$ with canonical dual $\tilde{\mathcal{E}}=\left\{\tilde{e}_{j}\right\}_{j \in G}$. Let $a: I \rightarrow G$ be an associated map.

(a) We say $(\mathcal{F}, a, \mathcal{E})$ has the weak dual HAP if for every $\varepsilon>0$, there is an integer $N_{\varepsilon}>0$ so that for every $i \in I$ we have

$$
\operatorname{dist}\left(f_{i}, \overline{\operatorname{span}}\left\{\tilde{e}_{j}: j \in S_{N_{\varepsilon}}(a(i))\right\}\right)<\varepsilon .
$$

(b) We say $(\mathcal{F}, a, \mathcal{E})$ has the strong dual HAP if for every $\varepsilon>0$, there is an integer $N_{\varepsilon}>0$ so that for every $i \in I$ we have

$$
\left\|f_{i}-\sum_{j \in S_{N_{\varepsilon}}(a(i))}\left\langle f_{i}, e_{j}\right\rangle \tilde{e}_{j}\right\|<\varepsilon .
$$

\subsection{Self-Localization}

It is also useful to consider localizations where the system $\mathcal{F}=\left\{f_{i}\right\}_{i \in I}$ is compared to itself or to its canonical dual frame instead of to a reference system $\mathcal{E}$. An analogous polynomial or exponential "intrinsic localization" was independently introduced by Gröchenig in [36]; see also $[33,38]$. Although there is no reference system, we still require a mapping $a: I \rightarrow G$ associating $I$ with a group $G$.

Definition 6 (Self-Localization). Let $\mathcal{F}=\left\{f_{i}\right\}_{i \in I}$ be a sequence in $H$, and let $a: I \rightarrow G$ be an associated map.

(a) We say that $(\mathcal{F}, a)$ is $\ell^{p}$-self-localized if there exists $r \in \ell^{p}(G)$ such that

$$
\forall i, j \in I, \quad\left|\left\langle f_{i}, f_{j}\right\rangle\right| \leq r_{a(i)-a(j)} .
$$

(b) If $\mathcal{F}$ is a frame sequence, then we say that $(\mathcal{F}, a)$ is $\ell^{p}$-localized with respect to its canonical dual frame sequence $\tilde{\mathcal{F}}=\left\{\tilde{f}_{i}\right\}_{i \in I}$ if there exists $r \in \ell^{p}(G)$ such that

$$
\forall i, j \in I, \quad\left|\left\langle f_{i}, \tilde{f}_{j}\right\rangle\right| \leq r_{a(i)-a(j)} .
$$

Remark 2. If $I=G$ and $a$ is the identity map, then $(\mathcal{F}, a)$ is $\ell^{1}$-self-localized if and only if $(\mathcal{F}, a, \mathcal{F})$ is $\ell^{1}$-localized. However, if $a$ is not the identity map, then this need not be the case. For example, every orthonormal basis is $\ell^{1}$-self-localized regardless of which map $a$ is chosen, but in Example 11 we construct an orthonormal basis $\mathcal{F}=\left\{f_{i}\right\}_{i \in \mathbf{Z}}$ and a map $a: \mathbf{Z} \rightarrow \mathbf{Z}$ such that $(\mathcal{F}, a, \mathcal{E})$ is not $\ell^{1}$-localized for any Riesz basis $\mathcal{E}$; in fact, $(\mathcal{F}, a, \mathcal{E})$ cannot even possess both $\ell^{2}$-column decay and $\ell^{2}$-row decay for any Riesz basis $\mathcal{E}$.

We show in Example 12 that $\ell^{1}$-localization with respect to the dual frame does not imply $\ell^{1}$-self-localization. However, the following result proved in Part I states that the converse is true. This result will play a key role in Section 4.3 for determining the properties of the canonical dual frame of an irregular Gabor frame. 
Theorem 1. Let $\mathcal{F}=\left\{f_{i}\right\}_{i \in I}$ be a frame for $H$, and let $a: I \rightarrow G$ be an associated map such that $D^{+}(I, a)<\infty$. Let $\tilde{\mathcal{F}}$ be the canonical dual frame and $S^{-1 / 2}(\mathcal{F})$ the canonical Parseval frame. If $(\mathcal{F}, a)$ is $\ell^{1}$-self-localized, then:

(a) $(\mathcal{F}, a)$ is $\ell^{1}$-localized with respect to its canonical dual frame $\tilde{\mathcal{F}}=\left\{\tilde{f}_{i}\right\}_{i \in I}$,

(b) $(\tilde{\mathcal{F}}, a)$ is $\ell^{1}$-self-localized, and

(c) $\left(S^{-1 / 2}(\mathcal{F}), a\right)$ is $\ell^{1}$-self-localized.

\subsection{Relative Measure}

\section{Definition 7.}

(a) Let $\mathcal{F}=\left\{f_{i}\right\}_{i \in I}$ and $\mathcal{E}=\left\{e_{j}\right\}_{j \in G}$ be frame sequences in $H$, and let $a: I \rightarrow G$ be an associated map. Let $P_{\mathcal{F}}, P_{\mathcal{E}}$ denote the orthogonal projections of $H$ onto $\overline{\operatorname{span}}(\mathcal{F})$ and $\overline{\operatorname{span}}(\mathcal{E})$, respectively. Then given a free ultrafilter $p$ and a sequence of centers $c=\left(c_{N}\right)_{N \in \mathbf{N}}$ in $G$, we define the relative measure of $\mathcal{F}$ with respect to $\mathcal{E}$, $p$, and $c$ to be

$$
\mathcal{M}_{\mathcal{E}}(\mathcal{F} ; p, c)=\lim _{N \in \mathbf{N}} \frac{1}{\left|I_{N}\left(c_{N}\right)\right|} \sum_{i \in I_{N}\left(c_{N}\right)}\left\langle P_{\mathcal{E}} f_{i}, \tilde{f}_{i}\right\rangle .
$$

The relative measure of $\mathcal{E}$ with respect to $\mathcal{F}, p$, and $c$ is

$$
\mathcal{M}_{\mathcal{F}}(\mathcal{E} ; p, c)=p-\lim _{N \in \mathbf{N}} \frac{1}{\left|S_{N}\left(c_{N}\right)\right|} \sum_{j \in S_{N}\left(c_{N}\right)}\left\langle P_{\mathcal{F}} \tilde{e}_{j}, e_{j}\right\rangle .
$$

(b) If $\overline{\operatorname{span}}(\mathcal{E}) \supset \overline{\operatorname{span}}(\mathcal{F})$ then $P_{\mathcal{E}}$ is the identity map and $\mathcal{E}$ plays no role in determining the value of $\mathcal{M}_{\mathcal{E}}(\mathcal{F} ; p, c)$. Therefore, in this case we define the measure of $\mathcal{F}$ with respect to $p$ and $c$ to be

$$
\mathcal{M}(\mathcal{F} ; p, c)=p-\lim _{N \in \mathbf{N}} \frac{1}{\left|I_{N}\left(c_{N}\right)\right|} \sum_{i \in I_{N}\left(c_{N}\right)}\left\langle f_{i}, \tilde{f}_{i}\right\rangle .
$$

Since $\left\langle f_{i}, \tilde{f}_{i}\right\rangle=\left\|S^{-1 / 2} f_{i}\right\|^{2}$, we have that $\mathcal{M}(\mathcal{F} ; p, c)$ is real. Additionally, since $S^{-1 / 2}(\mathcal{F})$ is a Parseval frame, we have $0 \leq\left\langle f_{i}, \tilde{f}_{i}\right\rangle \leq 1$ for all $i$, and therefore

$$
0 \leq \mathcal{M}(\mathcal{F} ; p, c) \leq 1
$$

For this case we further define the lower and upper measures of $\mathcal{F}$ by

$$
\begin{aligned}
& \mathcal{M}^{-}(\mathcal{F})=\liminf _{N \rightarrow \infty} \inf _{j \in G} \frac{1}{\left|I_{N}(j)\right|} \sum_{i \in I_{N}(j)}\left\langle f_{i}, \tilde{f}_{i}\right\rangle, \\
& \mathcal{M}^{+}(\mathcal{F})=\limsup _{N \rightarrow \infty} \sup _{j \in G} \frac{1}{\left|I_{N}(j)\right|} \sum_{i \in I_{N}(j)}\left\langle f_{i}, \tilde{f}_{i}\right\rangle .
\end{aligned}
$$

It can be seen that there exist free ultrafilters $p^{-}, p^{+}$and sequences of centers $c^{-}, c^{+}$such that $\mathcal{M}^{-}(\mathcal{F})=\mathcal{M}\left(\mathcal{F} ; p^{-}, c^{-}\right)$and $\mathcal{M}^{+}(\mathcal{F})=\mathcal{M}\left(\mathcal{E} ; p^{+}, c^{+}\right)$.

(c) When $\overline{\operatorname{span}}(\mathcal{F}) \supset \overline{\operatorname{span}}(\mathcal{E})$, we define the measures $\mathcal{M}(\mathcal{E} ; p, c)$ and $\mathcal{M}^{ \pm}(\mathcal{E})$ in an analogous manner.

Example 4. The following special cases show that the measure of a Riesz basis is 1. 
(a) If $\overline{\operatorname{span}}(\mathcal{E}) \supset \overline{\operatorname{span}}(\mathcal{F})$ and $\mathcal{F}$ is a Riesz sequence then $\left\langle f_{i}, \tilde{f}_{i}\right\rangle=1$ for every $i \in I$, so $\mathcal{M}(\mathcal{F} ; p, c)=\mathcal{M}^{+}(\mathcal{F})=\mathcal{M}^{-}(\mathcal{F})=1$.

(b) If $\overline{\operatorname{span}}(\mathcal{F}) \supset \overline{\operatorname{span}}(\mathcal{E})$ and $\mathcal{E}$ is a Riesz sequence then $\left\langle\tilde{e}_{j}, e_{j}\right\rangle=1$ for every $j \in G$, so $\mathcal{M}(\mathcal{E} ; p, c)=\mathcal{M}^{+}(\mathcal{E})=\mathcal{M}^{-}(\mathcal{E})=1$.

Example 5 (Lattice Gabor Systems). Consider a lattice Gabor frame, i.e., a frame of the form $\mathcal{G}\left(g, \alpha \mathbf{Z}^{d} \times \beta \mathbf{Z}^{d}\right)$. The canonical dual frame is a lattice Gabor frame of the form $\mathcal{G}\left(\tilde{g}, \alpha \mathbf{Z}^{d} \times \beta \mathbf{Z}^{d}\right)$ for some $\tilde{g} \in L^{2}\left(\mathbf{R}^{d}\right)$. By the Wexler-Raz relations, we have $\langle g, \tilde{g}\rangle=(\alpha \beta)^{d}$ (we also derive this fact directly from our results in Corollary 2). Since for all $k, n$ we have $\left\langle M_{\beta n} T_{\alpha k} g, M_{\beta n} T_{\alpha k} \tilde{g}\right\rangle=\langle g, \tilde{g}\rangle$, it follows that for any free ultrafilter $p$ and sequence of centers $c=\left(c_{N}\right)_{N \in \mathbf{N}}$ in $\alpha \mathbf{Z}^{d} \times \beta \mathbf{Z}^{d}$,

$$
\mathcal{M}\left(\mathcal{G}\left(g, \alpha \mathbf{Z}^{d} \times \beta \mathbf{Z}^{d}\right) ; p, c\right)=\mathcal{M}^{ \pm}\left(\mathcal{G}\left(g, \alpha \mathbf{Z}^{d} \times \beta \mathbf{Z}^{d}\right)\right)=\langle g, \tilde{g}\rangle=(\alpha \beta)^{d} .
$$

Since we also have $D_{B}^{ \pm}\left(\alpha \mathbf{Z}^{d} \times \beta \mathbf{Z}^{d}\right)=(\alpha \beta)^{-d}$, we conclude that

$$
\mathcal{M}^{ \pm}\left(\mathcal{G}\left(g, \alpha \mathbf{Z}^{d} \times \beta \mathbf{Z}^{d}\right)\right)=\frac{1}{D_{B}^{\mp}\left(\alpha \mathbf{Z}^{d} \times \beta \mathbf{Z}^{d}\right)} .
$$

Equation (2.6) is essentially a special case of the Density-Relative Measure Theorem of Part I, which states that if $\mathcal{F}, \mathcal{E}$ are frame sequences such that $(\mathcal{F}, a, \mathcal{E})$ has both $\ell^{2}$-column and row decay and $D^{+}(I, a)<\infty$, then

$$
\mathcal{M}_{\mathcal{F}}(\mathcal{E} ; p, c)=D(p, c) \cdot \mathcal{M}_{\mathcal{E}}(\mathcal{F} ; p, c)
$$

for every free ultrafilter $p$ and choice of centers $c=\left(c_{N}\right)_{N \in \mathbf{N}}$ in $G$. We apply this general result to irregular Gabor frames in Section 4.1.

\section{Localization of Gabor Systems}

In this section we will determine the localization properties of Gabor systems.

\subsection{Notation and Preliminary Observations for Gabor Systems Gabor Systems and the Reference System}

A generic Gabor system generated by a function $g \in L^{2}\left(\mathbf{R}^{d}\right)$ and a sequence $\Lambda \subset \mathbf{R}^{2 d}$ will be written in any of the following forms:

$$
\mathcal{G}(g, \Lambda)=\left\{M_{\omega} T_{x} g\right\}_{(x, \omega) \in \Lambda}=\left\{e^{2 \pi i \omega \cdot t} g(t-x)\right\}_{(x, \omega) \in \Lambda}=\left\{g_{\lambda}\right\}_{\lambda \in \Lambda} .
$$

In the case that $\mathcal{G}(g, \Lambda)$ is a frame sequence we let

$$
\tilde{\mathcal{G}}=\left\{\tilde{g}_{\lambda}\right\}_{\lambda \in \Lambda}
$$

denote the canonical dual frame sequence in $\overline{\operatorname{span}}(\mathcal{G}(g, \Lambda))$, but it is important to note that while $g_{\lambda}$ is a time-frequency shift of $g$, it need not be the case that the functions $\tilde{g}_{\lambda}$ are time-frequency shifts of a single function. We address the question of the structure of the dual frame in more detail in Section 4.3.

Our reference systems will be lattice Gabor systems indexed by the group

$$
G=\alpha \mathbf{Z}^{d} \times \beta \mathbf{Z}^{d},
$$


where $\alpha, \beta>0$ are fixed scalars. For compactness of notation, we usually let $G$ implicitly denote the group above, only writing out $\alpha \mathbf{Z}^{d} \times \beta \mathbf{Z}^{d}$ when we wish to explicitly emphasize the values of $\alpha, \beta$. Thus our reference systems have the form

$$
\mathcal{G}(\phi, G)=\mathcal{G}\left(\phi, \alpha \mathbf{Z}^{d} \times \beta \mathbf{Z}^{d}\right)=\left\{M_{\eta} T_{u} \phi\right\}_{(\eta, u) \in G}=\left\{M_{\beta n} T_{\alpha k} \phi\right\}_{k, n \in \mathbf{Z}^{d}} .
$$

The canonical dual frame of a lattice Gabor frame sequence is another lattice Gabor frame sequence $\mathcal{G}(\tilde{\phi}, G)$, generated by some dual window $\tilde{\phi} \in L^{2}\left(\mathbf{R}^{d}\right)$. Usually the reference system makes an appearance only during the course of a proof, and does not appear in the statement of most of the theorems.

\section{Cubes and the a Mapping}

For simplicity of notation, we introduce the following abbreviations.

Given $x=\left(x_{1}, \ldots, x_{d}\right) \in \mathbf{R}^{d}$, we define $\operatorname{Int}(x)=\left(\left\lfloor x_{1}\right\rfloor, \ldots,\left\lfloor x_{d}\right\rfloor\right)$ and $\operatorname{Frac}(x)=$ $x-\operatorname{Int}(x)$.

Let $\alpha, \beta$ be fixed. Then given a point $x=\left(x_{1}, \ldots, x_{d}\right) \in \mathbf{R}^{d}$, we set

$$
\bar{x}=\alpha \operatorname{Int}\left(\frac{x}{\alpha}\right), \quad \tilde{x}=\alpha \operatorname{Frac}\left(\frac{x}{\alpha}\right), \quad \bar{\omega}=\beta \operatorname{Int}\left(\frac{\omega}{\beta}\right), \quad \tilde{\omega}=\beta \operatorname{Frac}\left(\frac{\omega}{\beta}\right) .
$$

Note the implicit dependence on $\alpha$ and $\beta$ in this notation.

We define the map $a: \Lambda \rightarrow G$ as in Example 3 by rounding off to a near element of $G$, i.e.,

$$
a(x, \omega)=\left(\alpha \operatorname{Int}\left(\frac{x}{\alpha}\right), \beta \operatorname{Int}\left(\frac{\omega}{\beta}\right)\right)=(\bar{x}, \bar{\omega}), \quad(x, \omega) \in \Lambda .
$$

Given $z=(x, y) \in \mathbf{R}^{2 d}$, let $Q_{r}(z)=Q_{r}(x, y)$ denote the closed cube in $\mathbf{R}^{2 d}$ centered at $z$ with side length $r$. Then given $j \in G=\alpha \mathbf{Z}^{d} \times \beta \mathbf{Z}^{d}$, we have

$$
S_{N}(j)=G \cap Q_{N}(j) \quad \text { and } \quad I_{N}(j)=a^{-1}\left(G \cap Q_{N}(j)\right) .
$$

Note that $I_{N}(j)$ is very nearly $\Lambda \cap Q_{N}(j)$, except for the effect of rounding off points via the $a$ map. Thus

$$
\begin{aligned}
\left|S_{N}(j)\right| & =\left|G \cap Q_{N}(j)\right| \approx(\alpha \beta)^{-d} N^{2 d}, \\
\left|I_{N}(j)\right| & =\left|a^{-1}\left(G \cap Q_{N}(j)\right)\right| \approx\left|\Lambda \cap Q_{N}(j)\right| .
\end{aligned}
$$

\section{Density and Measure}

Recall from (2.4) the definitions of the lower and upper Beurling densities of the index set $\Lambda$ :

$$
D_{B}^{-}(\Lambda)=\liminf _{N \rightarrow \infty} \inf _{j \in \mathbf{R}^{2 d}} \frac{\left|\Lambda \cap Q_{N}(j)\right|}{N^{2 d}}
$$

and

$$
D_{B}^{+}(\Lambda)=\limsup _{N \rightarrow \infty} \sup _{j \in \mathbf{R}^{2 d}} \frac{\left|\Lambda \cap Q_{N}(j)\right|}{N^{2 d}} .
$$

Note that taking the inf and sup over $j \in G$ instead of $j \in \mathbf{R}^{2 d}$ does not affect the value of these densities. Example 2 derived the relationship between the Beurling densities $D_{B}^{ \pm}(\Lambda)$ and the densities $D^{ \pm}(\Lambda, a)$ defined in this article. Specifically,

$$
\begin{aligned}
D_{B}^{+}(\Lambda) & =(\alpha \beta)^{-d} D^{+}(\Lambda, a) \\
& =(\alpha \beta)^{-d} \limsup _{N \rightarrow \infty} \sup _{j \in \alpha \mathbf{Z}^{d} \times \beta \mathbf{Z}^{d}} \frac{\left|a^{-1}\left(G \cap Q_{N}(j)\right)\right|}{\left|G \cap Q_{N}(j)\right|},
\end{aligned}
$$


and similarly $D_{B}^{-}(\Lambda)=(\alpha \beta)^{-d} D^{-}(\Lambda, a)$. In light of these equations, we define the Beurling density of $\Lambda$ with respect to a free ultrafilter $p$ and a sequence of centers $c=\left(c_{N}\right)_{N \in \mathbf{N}}$ in $\mathbf{R}^{2 d}$ to be

$$
D_{B}(\Lambda ; p, c)=(\alpha \beta)^{-d} D(\Lambda, a ; p, c)=(\alpha \beta)^{-d} p \text {-lime } \frac{\left|a^{-1}\left(G \cap Q_{N}\left(c_{N}\right)\right)\right|}{\left|G \cap Q_{N}\left(c_{N}\right)\right|} .
$$

Our results for Gabor systems will all be stated in terms of these Beurling densities.

The measure of a Gabor frame sequence $\mathcal{G}(g, \Lambda)$ with respect to a free ultrafilter $p$ and a sequence of centers $c=\left(c_{N}\right)_{N \in \mathbf{N}}$ in $\mathbf{R}^{2 d}$ is

$$
\mathcal{M}(\mathcal{G}(g, \Lambda) ; p, c)=p \text { - } \lim _{N \in \mathbf{N}} \frac{1}{\left|a^{-1}\left(G \cap Q_{N}\left(c_{N}\right)\right)\right|} \sum_{a^{-1}\left(G \cap Q_{N}\left(c_{N}\right)\right)}\left\langle g_{\lambda}, \tilde{g}_{\lambda}\right\rangle .
$$

In particular, note that if $\mathcal{G}(g, \Lambda)$ is a Riesz sequence then $\left\langle g_{\lambda}, \tilde{g}_{\lambda}\right\rangle=1$ for all $\lambda$, so the measure is $\mathcal{M}^{ \pm}(\mathcal{G}(g, \Lambda))=1$. Also recall that the measure of a lattice Gabor system was computed in Example 5.

By making the approximations in (3.2) precise, the next lemma reformulates the density and measure in such a way that it becomes clear that they do not depend on the choice of $\alpha, \beta$. That is, the density of $I$ and the measure of $\mathcal{G}(g, \Lambda)$ are independent of the choice of reference group. Analogous reformulations of the upper and lower density and measures also hold under the same hypotheses.

Lemma 1. Let $\Lambda \subset \mathbf{R}^{2 d}$ be given.

(a) If $D_{B}^{+}(\Lambda)<\infty$, then for any free ultrafilter $p$ and any sequence of centers $c=$ $\left(c_{N}\right)_{N \in \mathbf{N}}$ in $\mathbf{R}^{2 d}$,

$$
D_{B}(\Lambda ; p, c)=p \text {-lim } \frac{\left|\Lambda \cap Q_{N}\left(c_{N}\right)\right|}{N^{2 d}} .
$$

(b) Let $g \in L^{2}\left(\mathbf{R}^{d}\right)$ be given. If $0<D_{B}^{-}(\Lambda) \leq D_{B}^{+}(\Lambda)<\infty$, then for any free ultrafilter $p$ and any sequence of centers $c=\left(c_{N}\right)_{N \in \mathbf{N}}$ in $\mathbf{R}^{2 d}$,

$$
\mathcal{M}(\mathcal{G}(g, \Lambda) ; p, c)=p-\lim _{N \in \mathbf{N}} \frac{1}{\left|\Lambda \cap Q_{N}\left(c_{N}\right)\right|} \sum_{\lambda \in \Lambda \cap Q_{N}\left(c_{N}\right)}\left\langle g_{\lambda}, \tilde{g}_{\lambda}\right\rangle .
$$

\section{Proof.}

(a) The map $a$ is a bounded perturbation of the identity map. In particular, if $\delta=$ $\max \{\alpha, \beta\}$, then we have

$$
\Lambda \cap Q_{N-\delta}\left(c_{N}\right) \subset a^{-1}\left(G \cap Q_{N}\left(c_{N}\right)\right) \subset \Lambda \cap Q_{N+\delta}\left(c_{N}\right) .
$$

Since the upper density is finite, there is a constant $C$ such that $\left|\Lambda \cap Q_{N+\delta}\left(c_{N}\right) \backslash Q_{N}\left(c_{n}\right)\right| \leq$ $C N^{2 d-1}$. Using the second inclusion in (3.3), we therefore have

$$
\begin{aligned}
D_{B}(\Lambda ; p, c) & =(\alpha \beta)^{-d} p_{N \in \mathbf{N}} \frac{\left|a^{-1}\left(G \cap Q_{N}\left(c_{N}\right)\right)\right|}{\left|G \cap Q_{N}\left(c_{N}\right)\right|} \\
& \leq(\alpha \beta)^{-d} p_{N \in \mathbf{N}} \frac{\left|\Lambda \cap Q_{N+\delta}\left(c_{N}\right)\right|}{\left|G \cap Q_{N}\left(c_{N}\right)\right|}
\end{aligned}
$$




$$
\begin{aligned}
& =(\alpha \beta)^{-d} p-\lim \left(\frac{\left|\Lambda \cap Q_{N}\left(c_{N}\right)\right|}{\left|G \cap Q_{N}\left(c_{N}\right)\right|}+\frac{\left|\Lambda \cap Q_{N+\delta}\left(c_{N}\right) \backslash Q_{N}\left(c_{N}\right)\right|}{\left|G \cap Q_{N}\left(c_{N}\right)\right|}\right) \\
& =p-\lim _{N \in \mathbf{N}} \frac{\left|\Lambda \cap Q_{N}\left(c_{N}\right)\right|}{N^{2 d}}
\end{aligned}
$$

and a similar computation making use of the first inclusion in (3.3) yields the opposite inequality.

(b) The fact that $0<D_{B}^{-}(\Lambda)$ and $D_{B}^{+}(\Lambda)<\infty$ implies that there exist $C_{1}, C_{2}>0$ such that for all $N$ large enough and all $j \in G$ we have $C_{1} N^{2 d} \leq\left|\Lambda \cap Q_{N}(j)\right| \leq C_{2} N^{2 d}$. Combining this with (3.3) then implies that

$$
p-\lim _{N \in \mathbf{N}} \frac{\left|\Lambda \cap Q_{N}\left(c_{N}\right)\right|}{\left|a^{-1}\left(G \cap Q_{N}\left(c_{N}\right)\right)\right|}=1 .
$$

Since $0 \leq\left\langle g_{\lambda}, \tilde{g}_{\lambda}\right\rangle \leq 1$, we therefore have

$$
\begin{aligned}
\mathcal{M}(\mathcal{G}(g, \Lambda) ; p, c) & =p-\lim _{N \in \mathbf{N}} \frac{1}{\left|a^{-1}\left(G \cap Q_{N}\left(c_{N}\right)\right)\right|} \sum_{\lambda \in a^{-1}\left(G \cap Q_{N}\left(c_{N}\right)\right)}\left\langle g_{\lambda}, \tilde{g}_{\lambda}\right\rangle \\
\leq & p-\lim _{N \in \mathbf{N}} \frac{1}{\left|\Lambda \cap Q_{N}\left(c_{N}\right)\right|} \sum_{\lambda \in \Lambda \cap Q_{N+\delta}\left(c_{N}\right)}\left\langle g_{\lambda}, \tilde{g}_{\lambda}\right\rangle \\
\leq & p \text {-lim } \frac{1}{\left|\Lambda \cap Q_{N}\left(c_{N}\right)\right|} \sum_{\lambda \in \Lambda \cap Q_{N}\left(c_{N}\right)}\left\langle g_{\lambda}, \tilde{g}_{\lambda}\right\rangle \\
& +p-\lim _{N \in \mathbf{N}} \frac{\left|\Lambda \cap Q_{N+\delta}\left(c_{N}\right) \backslash Q_{N}\left(c_{N}\right)\right|}{\left|\Lambda \cap Q_{N}\left(c_{N}\right)\right|}
\end{aligned}
$$

and the final term in this computation is zero. Combining this with a similar computation for the opposite inequality then yields the result.

\subsection{Localization with Respect to the Box Function}

We now show that any Gabor system $\mathcal{G}(g, \Lambda)$ has $\ell^{2}$-row decay with respect to the Gabor orthonormal basis $\mathcal{G}\left(\chi, \mathbf{Z}^{2 d}\right)$ generated by the "box function" $\chi=\chi_{\left[-\frac{1}{2}, \frac{1}{2}\right)^{d}}$, and that we recover as a consequence the fact first proved in [14] that any Gabor system that forms a Bessel sequence must have finite density.

The Gabor system generated by the box function is convenient both because it is an orthonormal basis and because the index set is $G=\mathbf{Z}^{2 d}$ (so, in particular, $\alpha=\beta=$ 1). However, in general this is not a useful basis in applications because the generating function $\chi$ is poorly concentrated in the time-frequency plane (in fact, by the Balian-Low Theorem, no Gabor Riesz basis of the form $\mathcal{G}\left(\phi, \alpha \mathbf{Z}^{d} \times \beta \mathbf{Z}^{d}\right)$ can have a generator $\phi$ that is simultaneously well-concentrated in both time and frequency). In the next section we show in more detail how time-frequency concentration is related to localization.

Proposition 1. If $g \in L^{2}\left(\mathbf{R}^{d}\right)$ and $\Lambda \subset \mathbf{R}^{2 d}$, then $\left(\mathcal{G}(g, \Lambda), a, \mathcal{G}\left(\chi, \mathbf{Z}^{2 d}\right)\right)$ has $\ell^{2}$-row decay.

Proof. Choose $\varepsilon>0$, and let $R>0$ be large enough that

$$
\int_{\mathbf{R}^{d} \backslash[-R, R]^{d}}|g(t)|^{2} d t<\varepsilon
$$


Fix an even integer $N_{\varepsilon}>R+3$.

Consider now any $(x, \omega) \in \Lambda$. Since $\alpha=\beta=1$, we have $\tilde{x}=\operatorname{Frac}(x) \in[0,1)^{d}$ and $\tilde{\omega}=\operatorname{Frac}(\omega) \in[0,1)^{d}$. Note that for each $k \in \mathbf{Z}^{d},\left\{M_{n} T_{k} \chi\right\}_{n \in \mathbf{Z}^{d}}$ is an orthonormal basis for the subspace of $L^{2}\left(\mathbf{R}^{d}\right)$ consisting of functions supported in the unit cube $B_{k}$ in $\mathbf{R}^{d}$ centered at $k$. Therefore,

$$
\begin{aligned}
\sum_{(k, n) \in \mathbf{Z}^{2 d} \backslash S_{N_{\varepsilon}}(a(x, \omega))}\left|\left\langle M_{\omega} T_{x} g, M_{n} T_{k} \chi\right\rangle\right|^{2} & =\sum_{(k, n) \in \mathbf{Z}^{2 d} \backslash S_{N_{\varepsilon}}(\bar{x}, \bar{\omega})}\left|\left\langle M_{\tilde{\omega}} T_{\tilde{x}} g, M_{n-\bar{\omega}} T_{k-\bar{x}} \chi\right\rangle\right|^{2} \\
& =\sum_{(k, n) \in \mathbf{Z}^{2 d} \backslash S_{N_{\varepsilon}}(0,0)}\left|\left\langle M_{\tilde{\omega}} T_{\tilde{x}} g, M_{n} T_{k} \chi\right\rangle\right|^{2} \\
& \leq \sum_{k \in \mathbf{Z}^{d} \backslash\left[-\frac{N_{\varepsilon}}{2}, \frac{N_{\varepsilon}}{2}\right)^{d}} \sum_{n \in \mathbf{Z}^{d}}\left|\left\langle M_{\tilde{\omega}} T_{\tilde{x}} g, M_{n} T_{k} \chi\right\rangle\right|^{2} \\
& =\sum_{k \in \mathbf{Z}^{d} \backslash\left[-\frac{N_{\varepsilon}}{2}, \frac{N_{\varepsilon}}{2}\right)^{d}} \int_{B_{k}}\left|M_{\tilde{\omega}} T_{\tilde{x}} g(t)\right|^{2} d t \\
& \leq \int_{\mathbf{R}^{d} \backslash\left[\frac{-N_{\varepsilon}+1}{2}, \frac{N_{\varepsilon}-1}{2}\right]^{d}}|g(t-\tilde{x})|^{2} d t \\
& \leq \int_{\mathbf{R}^{d} \backslash\left[-\frac{R}{2}, \frac{R}{2}\right]^{d}}|g(t)|^{2} d t<\varepsilon .
\end{aligned}
$$

Note that the preceding result does not require that $\Lambda$ have finite density. However, we next observe that it follows as a consequence of Part I results that the density must be finite if the Gabor system is a Bessel sequence.

Corollary 1. If $g \in L^{2}\left(\mathbf{R}^{d}\right)$ and $\Lambda \subset \mathbf{R}^{2 d}$ are such that $\mathcal{G}(g, \Lambda)$ is a Bessel sequence, then $D_{B}^{+}(\Lambda)<\infty$.

Proof. Since $\left(\mathcal{G}(g, \Lambda), a, \mathcal{G}\left(\chi, \mathbf{Z}^{2 d}\right)\right)$ has $\ell^{2}$-row decay and $\mathcal{G}(g, \Lambda)$ is norm-bounded below, all the hypotheses of $[4$, Theorem 4$]$ are fulfilled, and consequently the upper density must be finite.

\subsection{Localization with Respect to $M^{1}$ Functions}

For most applications in time-frequency analysis, the generator of a Gabor system must possess some amount of joint concentration in both time and frequency. Concentration is quantified by the norms of the modulation spaces, which are the Banach function spaces naturally associated to time-frequency analysis. The modulation spaces were invented and extensively investigated by Feichtinger, with some of the main references being [22, 24, 29, $30,31,27]$. For a detailed development of the theory of modulation spaces and their weighted counterparts, we refer to the original literature mentioned above and to [35, Chapters 11$13]$.

For our purposes, the following special case of unweighted modulation spaces will be sufficient. 


\section{Definition 8.}

(a) The Short-Time Fourier Transform (STFT) of a tempered distribution $g \in \mathcal{S}^{\prime}\left(\mathbf{R}^{d}\right)$ with respect to a window function $\phi \in \mathcal{S}\left(\mathbf{R}^{d}\right)$ is

$$
V_{\phi} g(x, \omega)=\left\langle g, M_{\omega} T_{x} \phi\right\rangle, \quad(x, \omega) \in \mathbf{R}^{2 d} .
$$

(b) Let $\gamma(x)=2^{d / 4} e^{-\pi x \cdot x}$ be the Gaussian function, which has been normalized so that $\|\gamma\|_{2}=1$. Then for $1 \leq p \leq \infty$, the modulation space $M^{p}\left(\mathbf{R}^{d}\right)$ consists of all tempered distributions $f \in \mathcal{S}^{\prime}\left(\mathbf{R}^{d}\right)$ such that

$$
\|f\|_{M^{p}}=\left\|V_{\gamma} f\right\|_{L^{p}}=\left(\iint_{\mathbf{R}^{2 d}}\left|\left\langle f, M_{\omega} T_{x} \gamma\right\rangle\right|^{p} d x d \omega\right)^{1 / p}<\infty
$$

with the usual adjustment if $p=\infty$.

$M^{p}$ is a Banach space for each $1 \leq p \leq \infty$, and any nonzero function $g \in M^{1}$ can be substituted for $\gamma$ in (3.4) to define an equivalent norm for $M^{p}$. We have $M^{2}=L^{2}$, and $\mathcal{S} \subsetneq M^{p} \subsetneq M^{q} \subsetneq \mathcal{S}^{\prime}$ for $1 \leq p<q \leq \infty$, where $\mathcal{S}$ is the Schwartz class. The box function $\chi$ lies in $M^{p}$ for $p>1$, but is not in $M^{1}$.

Remark 3. There is a complete characterization of the frame properties of lattice Gabor systems generated by the Gaussian:

(a) $\mathcal{G}\left(\gamma, \alpha \mathbf{Z}^{d} \times \beta \mathbf{Z}^{d}\right)$ is a frame for $L^{2}\left(\mathbf{R}^{d}\right)$ if $0<\alpha \beta<1$,

(b) $\mathcal{G}\left(\gamma, \alpha \mathbf{Z}^{d} \times \beta \mathbf{Z}^{d}\right)$ is a Riesz sequence in $L^{2}\left(\mathbf{R}^{d}\right)$ if $\alpha \beta>1$ (but not a Riesz basis),

(c) $\mathcal{G}\left(\gamma, \alpha \mathbf{Z}^{d} \times \beta \mathbf{Z}^{d}\right)$ is complete but not a frame for $L^{2}\left(\mathbf{R}^{d}\right)$ if $\alpha \beta=1$.

Part (a) was proved in [54, 58, 59]; see also the simple proof given in [47]. Part (b) is a consequence of part (a) and the Wexler-Raz relations, and part (c) is easy to show using Zak transform techniques, see [35]. Such a complete characterization of frame properties is known for only a few specific functions [47, 50, 51], cf. also [12]. In particular such a characterization is not available for general $M^{1}$ functions. On the other hand, given any particular choice of $\alpha, \beta$ with $0<\alpha \beta<1$, it is easy to construct a function $g \in C_{c}^{\infty}\left(\mathbf{R}^{d}\right)$ such that $\mathcal{G}\left(g, \alpha \mathbf{Z}^{d} \times \beta \mathbf{Z}^{d}\right)$ is a Parseval frame for $L^{2}\left(\mathbf{R}^{d}\right)$, cf. [18]. It is known that if $\mathcal{G}\left(\phi, \alpha \mathbf{Z}^{d} \times \beta \mathbf{Z}^{d}\right)$ is a frame for $L^{2}\left(\mathbf{R}^{d}\right)$ generated by a function $\phi \in M^{1}$, then the canonical dual frame $\mathcal{G}\left(\tilde{\phi}, \alpha \mathbf{Z}^{d} \times \beta \mathbf{Z}^{d}\right)$ has a generator $\tilde{\phi}$ that also lies in $M^{1}$ [40]. In Section 4.3 we will extend that result to the more general setting of irregular Gabor frames.

In addition to the modulation spaces, we will also need a special case of the Wiener amalgam spaces on $\mathbf{R}^{2 d}$. Feichtinger has developed a general notion of amalgam spaces, e.g., $[21,28,23,25,26]$. For an introduction (with extensive references to the original literature) to the particular Wiener amalgams appearing in the following definition, we refer to [43].

Definition 9. Given $1 \leq p \leq \infty$, the Wiener amalgam $W\left(\mathcal{C}, \ell^{p}\right)$ consists of all continuous functions $F$ on $\mathbf{R}^{2 d}$ for which

$$
\|F\|_{W\left(\mathcal{C}, \ell^{p}\right)}=\left(\sum_{(k, n) \in \mathbf{Z}^{2 d}} \sup _{(u, \eta) \in \mathbf{Q}_{\alpha, \beta}(\alpha k, \beta n)}|F(u, \eta)|^{p}\right)^{1 / p}<\infty,
$$

with the usual adjustment if $p=\infty$, and where

$$
\mathbf{Q}_{\alpha, \beta}(x, y)=[0, \alpha)^{d} \times[0, \beta)^{d}+(x, y) .
$$


$W\left(\mathcal{C}, \ell^{p}\right)$ is a Banach space, and its definition is independent of the values of $\alpha$ and $\beta$ in the sense that each choice of $\alpha, \beta$ yields an equivalent norm for $W\left(\mathcal{C}, \ell^{p}\right)$.

We will need the following lemma on the basic properties of the STFT. Part (a) is proved in [35, Theorem 12.2.1], and part (b) in [35, Lemma 12.1.1].

\section{Lemma 2.}

(a) Let $1 \leq p \leq \infty$ be given. If $g \in M^{p}$ and $\phi \in M^{1}$, then $V_{\phi} g \in W\left(\mathcal{C}, \ell^{p}\right)$, and

$$
\left\|V_{\phi} g\right\|_{W\left(\mathcal{C}, \ell^{p}\right)} \leq C\|g\|_{M^{p}}\|\phi\|_{M^{1}}
$$

where $C$ is a constant independent of $g$ and $\phi$.

(b) Let $f, g \in L^{2}\left(\mathbf{R}^{d}\right)$ be given. If $V_{g} f \in L^{1}\left(\mathbf{R}^{2 d}\right)$, then $f, g \in M^{1}\left(\mathbf{R}^{d}\right)$.

Next we will show that if the generator of our reference system is an $M^{1}$ function $\phi$, then $(\mathcal{G}(g, \Lambda), a, \mathcal{G}(\phi, G))$ is $\ell^{p}$-localized whenever $g \in M^{p}$. We also show that the converse is true if we assume that $\phi$ generates a frame. Thus the degree of localization is tied to the time-frequency concentration of the generator $g$. This is a stronger statement than previously known results, which only demonstrated that Gabor frames satisfy the weak HAP with respect to reference systems generated by the Gaussian. We will also show that if $g \in M^{1}$ then $(\mathcal{G}(g, \Lambda), a)$ is $\ell^{1}$-self-localized (this statement does not require a reference system). However, the converse of this is false. For example, if we set $g=\chi$ and $\Lambda=\mathbf{Z}^{2 d}$, then $\mathcal{G}(g, \Lambda)$ is an orthonormal basis for $L^{2}\left(\mathbf{R}^{d}\right)$ and hence is $\ell^{1}$-self-localized, but $g \notin M^{1}$.

Theorem 2. Let $g \in L^{2}\left(\mathbf{R}^{d}\right)$ and $\Lambda \subset \mathbf{R}^{2 d}$ be given. Let $\phi \in L^{2}\left(\mathbf{R}^{d}\right)$ and $\alpha, \beta>0$ be given, and fix $1 \leq p \leq 2$. Then the following statements hold.

(a) If $g \in M^{p}$ and $\phi \in M^{1}$ then $(\mathcal{G}(g, \Lambda), a, \mathcal{G}(\phi, G))$ is $\ell^{p}$-localized.

(b) Suppose $\phi \in M^{1}$ and $\alpha, \beta>0$ are such that $\mathcal{G}(\phi, G)$ is a frame for $L^{2}\left(\mathbf{R}^{d}\right)$. If $(\mathcal{G}(g, \Lambda), a, \mathcal{G}(\phi, G))$ is $\ell^{p}$-localized, then $g \in M^{p}$.

(c) If $g \in M^{1}$ and $\phi \in M^{p}$ then $(\mathcal{G}(g, \Lambda), a, \mathcal{G}(\phi, G))$ is $\ell^{p}$-localized.

(d) If $g \in M^{1}$ then $(\mathcal{G}(g, \Lambda), a)$ is $\ell^{1}$-self-localized.

Proof.

(a) Set

$$
r_{(\alpha k, \beta n)}=\sup _{(u, \eta) \in \mathbf{Q}_{\alpha, \beta}(\alpha k, \beta n)}\left|V_{\phi} g(-u,-\eta)\right| .
$$

By Lemma 2 we have $V_{\phi} g \in W\left(\mathcal{C}, \ell^{p}\right)$, so $r=\left(r_{(\alpha k, \beta n)}\right)_{(\alpha k, \beta n) \in G} \in \ell^{p}(G)$. Let $(x, \omega) \in \Lambda$ and $(u, v) \in G$ be given. Then, recalling the notations $\tilde{x}, \tilde{\omega}$ introduced in (3.1), since $\tilde{x} \in[0, \alpha)^{d}$ and $\tilde{\omega} \in[0, \beta)^{d}$, we have

$$
\begin{aligned}
& \left|\left\langle M_{\omega} T_{x} g, M_{v} T_{u} \phi\right\rangle\right|=\left|\left\langle g, M_{v-\bar{\omega}-\tilde{\omega}} T_{u-\bar{x}-\tilde{x}} \phi\right\rangle\right| \\
& =\left|V_{\phi} g(u-\bar{x}-\tilde{x}, v-\bar{\omega}-\tilde{\omega})\right| \leq r_{a(x, \omega)-(u, v)},
\end{aligned}
$$

so $\ell^{p}$-localization holds.

(b) By [40] or Theorem 7 , the dual frame of $\mathcal{G}(\phi, G)$ is a lattice Gabor frame $\mathcal{G}(\tilde{\phi}, G)$ with a dual window $\tilde{\phi} \in M^{1}$. Fix any $(x, \omega) \in \Lambda$. Expanding $M_{\omega} T_{x} g$ with respect to this frame, we have

$$
M_{\omega} T_{x} g=\sum_{k, n \in \mathbf{Z}^{d}}\left\langle M_{\omega} T_{x} g, M_{\beta n} T_{\alpha k} \phi\right\rangle M_{\beta n} T_{\alpha k} \tilde{\phi}
$$


with convergence in $L^{2}\left(\mathbf{R}^{d}\right)$. Now, by definition of $\ell^{p}$-localization, there exists $r \in \ell^{p}(G)$ such that

$$
\left|\left\langle M_{\omega} T_{x} g, M_{\beta n} T_{\alpha k} \phi\right\rangle\right| \leq r_{a(x, \omega)-(\alpha k, \beta n)}, \quad(\alpha k, \beta n) \in G .
$$

Consequently, $\left\{\left\langle M_{\omega} T_{x} g, M_{\beta n} T_{\alpha k} \phi\right\rangle\right\}_{k, n \in \mathbf{Z}^{d}} \in \ell^{p}$, and so by [35, Theorem 12.2.4] the series on the right-hand side of (3.5) converges in $M^{p}$-norm. Since it also converges in $L^{2}$-norm, the series must converge in $M^{p}$-norm to $M_{\omega} T_{x} g$, so $M_{\omega} T_{x} g \in M^{p}$. Since $M^{p}$ is closed under time-frequency shifts, we conclude $g \in M^{p}$.

(c) Since $\left|V_{\phi} g(x, \omega)\right|=\left|V_{g} \phi(-x,-\omega)\right|$, this follows from part (a).

(d) This can be shown directly, similarly to part (a), or by applying [4, Lemma 3] to part (a), using as a reference system any lattice Gabor frame $\mathcal{G}(\phi, G)$ whose generator $\phi$ lies in $M^{1}$.

\section{New Implications for Gabor Frames}

\subsection{Density and Overcompleteness for Gabor Systems}

Parts (b) and (c) of the following theorem are new results for Gabor frames and give a new interpretation of the density of a Gabor frame. Part (a) recovers the known density facts for irregular Gabor frames. These results are stated in terms of Beurling density, which was discussed in Section 3.1.

Theorem 3. Let $g \in L^{2}\left(\mathbf{R}^{d}\right)$ and $\Lambda \subset \mathbf{R}^{2 d}$ be given. If $\mathcal{G}(g, \Lambda)$ is a Gabor frame for $L^{2}\left(\mathbf{R}^{d}\right)$ then the following statements hold.

(a) $1 \leq D_{B}^{-}(\Lambda) \leq D_{B}^{+}(\Lambda)<\infty$.

(b) For any free ultrafilter $p$ and any sequence of centers $c=\left(c_{N}\right)_{N \in \mathbf{N}}$ in $\mathbf{R}^{2 d}$, we have

$$
\mathcal{M}(\mathcal{G}(g, \Lambda) ; p, c)=\frac{1}{D_{B}(\Lambda ; p, c)}
$$

and consequently

$$
\begin{gathered}
p-\lim \frac{1}{N_{N} \in \mathbb{N}} \sum_{\lambda \in \Lambda \cap Q_{N}\left(c_{N}\right)}\left\langle g_{\lambda}, \tilde{g}_{\lambda}\right\rangle=1 . \\
\text { (c) } \mathcal{M}^{-}(\mathcal{G}(g, \Lambda))=\frac{1}{D_{B}^{+}(\Lambda)} \text { and } \mathcal{M}^{+}(\mathcal{G}(g, \Lambda))=\frac{1}{D_{B}^{-}(\Lambda)} .
\end{gathered}
$$

\section{Proof.}

(a) In this part we use a reference system $\mathcal{G}(\gamma, G)$ generated by the Gaussian $\gamma$. If we take any $\alpha, \beta>0$ so that $\alpha \beta>1$, then $\mathcal{G}(\gamma, G)$ is a Riesz sequence in $L^{2}\left(\mathbf{R}^{d}\right)$, and $(\mathcal{G}(g, \Lambda), a, \mathcal{G}(\gamma, G))$ is $\ell^{2}$-localized by Theorem 2 . Therefore, we have by [4, Theorem 3] that $(\alpha \beta)^{d} D_{B}^{-}(\Lambda)=D^{-}(\Lambda, a) \geq 1$. Since this is true for any $\alpha \beta>1$, we conclude that $D_{B}^{-}(\Lambda) \geq 1$. The fact that $D_{B}^{+}(\Lambda)<\infty$ follows from Corollary 1 .

(b) In this part our reference system will be generated by the function

$$
\phi(x)=\prod_{k=1}^{d} \frac{e^{2 \pi i x_{k}}+1}{2} \chi_{\left[-\frac{1}{2}, \frac{1}{2}\right]}\left(x_{k}\right), \quad x=\left(x_{1}, \ldots, x_{d}\right) \in \mathbf{R}^{d} .
$$


Since $\phi$ is compactly supported, $\phi \in L^{1}\left(\mathbf{R}^{d}\right)$, and $\hat{\phi}(\omega)=\prod_{k=1}^{d} \frac{\sin \pi \omega_{k}}{2 \pi\left(\omega_{k}-\omega_{k}^{2}\right)} \in L^{1}\left(\mathbf{R}^{d}\right)$, it follows from [32, Theorem 3.2.17] that $\phi \in M^{1}$. If we set $\alpha=1 / 2$ and $\beta=1$, then since $\sum\left|\phi\left(x-\frac{1}{2} k\right)\right|^{2}=1$, we have by [35, Theorem 6.4.1] that $\mathcal{G}(\phi, G)$ is a Parseval frame for $L^{2}\left(\mathbf{R}^{d}\right)$. By direct computation, the measure of this frame is $\mathcal{M}(\mathcal{G}(\phi, G) ; p, c)=\|\phi\|_{2}^{2}=$ $2^{-d}$.

Since $(\mathcal{G}(g, \Lambda), a, \mathcal{G}(\phi, G))$ is $\ell^{2}$-localized, we have by [4, Theorem $\left.6(\mathrm{a})\right]$ that

$$
\mathcal{M}(\mathcal{G}(g, \Lambda) ; p, c)=\frac{\mathcal{M}(\mathcal{G}(\phi, G) ; p, c)}{D(p, c)}=\frac{2^{-d}}{2^{-d} D_{B}(\Lambda ; p, c)}=\frac{1}{D_{B}(\Lambda ; p, c)}
$$

This proves equation (4.1). Finally, the reformulation in (4.2) follows by applying Lemma 1 to $(4.1)$.

(c) Using the same reference system as in part (b), we have by [4, Theorem $6(\mathrm{a})]$ that

$$
2^{-d}=\mathcal{M}^{-}(\mathcal{G}(\phi, G)) \leq D^{+}(\Lambda, a) \cdot \mathcal{M}^{-}(\mathcal{G}(g, \Lambda)) \leq \mathcal{M}^{+}(\mathcal{G}(\phi, G))=2^{-d},
$$

so

$$
\mathcal{M}^{-}(\mathcal{G}(g, \Lambda))=\frac{2^{-d}}{D^{+}(\Lambda, a)}=\frac{1}{D_{B}^{+}(\Lambda)},
$$

and similarly $\mathcal{M}^{+}(\mathcal{G}(g, \Lambda))=\frac{1}{D_{B}^{-}(\Lambda)}$.

The Wexler-Raz relations are a remarkable set of equalities that hold for lattice Gabor frames, see $[19,48,57]$. There is no known analogue of the Wexler-Raz relations for arbitrary, irregular Gabor frames, so it is interesting that if we apply Theorem 3 to lattice Gabor frames, we recover the following special case of the Wexler-Raz relations.

Corollary 2. Assume that $g \in L^{2}\left(\mathbf{R}^{d}\right)$ and $\alpha, \beta>0$ are such that $\mathcal{G}\left(g, \alpha \mathbf{Z}^{d} \times \beta \mathbf{Z}^{d}\right)$ is a frame for $L^{2}\left(\mathbf{R}^{d}\right)$. Let $\mathcal{G}\left(\tilde{g}, \alpha \mathbf{Z}^{d} \times \beta \mathbf{Z}^{d}\right)$ be the canonical dual frame. Then $0<\alpha \beta \leq 1$ and

$$
\langle g, \tilde{g}\rangle=(\alpha \beta)^{d} .
$$

Proof. For a lattice, we have that $D_{B}^{ \pm}\left(\alpha \mathbf{Z}^{d} \times \beta \mathbf{Z}^{d}\right)=(\alpha \beta)^{-d}$ and that $\mathcal{M}^{ \pm}(\mathcal{G}(g, \Lambda))=$ $\langle g, \tilde{g}\rangle$. Therefore the result follows from parts (a) and (c) of Theorem 3.

We have the following complementary density relations for the case of Riesz sequences. While part (b) of the following result is known, part (a) is a new result for Gabor systems.

Theorem 4. Let $g \in L^{2}\left(\mathbf{R}^{d}\right)$ and $\Lambda \subset \mathbf{R}^{2 d}$ be given. If $\mathcal{G}(g, \Lambda)$ is a Riesz sequence in $L^{2}\left(\mathbf{R}^{d}\right)$ then the following statements hold.

(a) $0 \leq D_{B}^{-}(\Lambda) \leq D_{B}^{+}(\Lambda) \leq 1$.

(b) If $\mathcal{G}(g, \Lambda)$ is a Riesz basis for $L^{2}\left(\mathbf{R}^{d}\right)$, then $D_{B}^{-}(\Lambda)=D_{B}^{+}(\Lambda)=1$.

Proof.

(a) We use a reference system $\mathcal{G}(\gamma, G)$ generated by the Gaussian $\gamma$. If we take any $\alpha, \beta>0$ so that $\alpha \beta<1$, then $\mathcal{G}(\gamma, G)$ is a frame for $L^{2}\left(\mathbf{R}^{d}\right)$, and $(\mathcal{G}(g, \Lambda), a, \mathcal{G}(\gamma, G))$ is $\ell^{2}$-localized by Theorem 2. Therefore, we have by $\left[4\right.$, Theorem 3] that $(\alpha \beta)^{d} D_{B}^{+}(\Lambda)=D^{+}(\Lambda, a) \leq 1$. Since this is true for any $\alpha \beta<1$, we conclude that $D_{B}^{-}(\Lambda) \leq 1$.

(b) A Riesz basis is both a frame and a Riesz sequence, so this follows by combining part (a) 
with Theorem 3(a). Alternatively, if $\mathcal{G}(g, \Lambda)$ is a Riesz basis then $\mathcal{M}^{ \pm}(\mathcal{G}(g, \Lambda))=1$, so the result follows from Theorem 3(c).

Remark 4. We can also give an interpretation of the Beurling density of a Riesz sequence in terms of a measure of a frame. If $\mathcal{G}(g, \Lambda)$ is a Riesz sequence, then we have of course that $\mathcal{M}^{ \pm}(\mathcal{G}(g, \Lambda))=1$. However, since the closed span of $\mathcal{G}(g, \Lambda)$ is not all of $L^{2}\left(\mathbf{R}^{d}\right)$, when we apply the Density-Relative Measure Theorem of [4], we must deal with relative measures instead of measures. To be precise, suppose that

$$
\mathcal{G}(\phi, G)=\mathcal{G}\left(\phi, \alpha \mathbf{Z}^{d} \times \beta \mathbf{Z}^{d}\right)=\left\{\phi_{\mu}\right\}_{\mu \in G}
$$

is any Gabor frame for $L^{2}\left(\mathbf{R}^{d}\right)$ such that $\phi \in M^{1}\left(\mathbf{R}^{d}\right)$. Then we know that $(\mathcal{G}(g, \Lambda)$, $a, \mathcal{G}(\gamma, G))$ is $\ell^{2}$-localized. Let $\mathcal{G}(\tilde{\phi}, G)=\left\{\tilde{\phi}_{\mu}\right\}_{\mu \in G}$ be the canonical dual frame, and let $P_{g}$ denote the orthogonal projection of $L^{2}\left(\mathbf{R}^{d}\right)$ onto $\overline{\operatorname{span}}(\mathcal{G}(g, \Lambda))$. Let $p$ be any free ultrafilter and choose any sequence of centers $c=\left(c_{N}\right)_{N \in \mathbf{N}}$ in $\mathbf{R}^{2 d}$. Then [4, Theorem 5] implies that the following relation holds:

$$
\mathcal{M}_{\mathcal{G}(g, \Lambda)}(\mathcal{G}(\phi, G) ; p, c)=D(\Lambda ; p, c) \cdot \mathcal{M}(\mathcal{G}(g, \Lambda) ; p, c),
$$

where we have as before that

$$
D(\Lambda ; p, d)=(\alpha \beta)^{d} D_{B}(\Lambda ; p, c),
$$

and that

$$
\mathcal{M}(\mathcal{G}(g, \Lambda) ; p, c)=1
$$

since $\mathcal{G}(g, \Lambda)$ is a Riesz sequence. The remaining quantity in (4.3) is the relative measure of $\mathcal{G}(\phi, G)$ with respect to $\mathcal{G}(g, \Lambda)$, which is given by

$$
\mathcal{M}_{\mathcal{G}(g, \Lambda)}(\mathcal{G}(\phi, G) ; p, c)=p-\lim _{N \in \mathbf{N}} \frac{1}{\left|G \cap Q_{N}\left(c_{N}\right)\right|} \sum_{\mu \in G \cap Q_{N}\left(c_{N}\right)}\left\langle P_{g} \tilde{\phi}_{\mu}, \phi_{\mu}\right\rangle .
$$

Thus, we have the relationship

$$
\mathcal{M}_{\mathcal{G}(g, \Lambda)}(\mathcal{G}(\phi, G) ; p, c)=(\alpha \beta)^{d} D_{B}(\Lambda ; p, c),
$$

for every lattice Gabor frame $\mathcal{G}(\phi, G)$ with generator $\phi \in M^{1}$, every free ultrafilter $p$, and every choice of centers $c$.

Next we prove results on the relationship between the density, frame bounds, and norm of the generator of a Gabor frame.

Theorem 5. Let $g \in L^{2}\left(\mathbf{R}^{d}\right)$ and $\Lambda \subset \mathbf{R}^{2 d}$ be such that $\mathcal{G}(g, \Lambda)$ is a Gabor frame for $L^{2}\left(\mathbf{R}^{d}\right)$, with frame bounds $A, B$. Then the following statements hold.

(a) $A \leq D_{B}^{-}(\Lambda)\|g\|_{2}^{2} \leq D_{B}^{+}(\Lambda)\|g\|_{2}^{2} \leq B$.

(b) If $\mathcal{G}(g, \Lambda)$ is a tight frame, then $\Lambda$ has uniform Beurling density, that is, $D_{B}^{-}(\Lambda)=$ $D_{B}^{+}(\Lambda)$, and furthermore $A=D_{B}^{ \pm}(\Lambda)\|g\|_{2}^{2}$.

Proof. For a reference system, fix any $\phi \in M^{1}$ and any $\alpha, \beta>0$ such that $\mathcal{G}(\phi, G)$ is a Parseval frame for $L^{2}\left(\mathbf{R}^{d}\right)$. By Theorem $3(\mathrm{e})$ we have $\|\phi\|_{2}^{2}=(\alpha \beta)^{d}$. Further, $(\mathcal{G}(g, \Lambda), a, \mathcal{G}(\phi, G))$ is $\ell^{2}$-localized by Theorem 2, so [4, Theorem $\left.7(\mathrm{c})\right]$ implies that

$$
\begin{aligned}
\frac{A(\alpha \beta)^{d}}{\|g\|_{2}^{2}}=\frac{A\|\phi\|_{2}^{2}}{\|g\|_{2}^{2}} & \leq D^{-}(\Lambda, a) \\
& =(\alpha \beta)^{d} D_{B}^{-}(\Lambda)
\end{aligned}
$$




$$
\begin{aligned}
& \leq(\alpha \beta)^{d} D_{B}^{+}(\Lambda) \\
& =D^{+}(\Lambda, a)=\frac{B\|\phi\|_{2}^{2}}{\|g\|_{2}^{2}} \leq \frac{B(\alpha \beta)^{d}}{\|g\|_{2}^{2}}
\end{aligned}
$$

so part (a) follows. Part (b) is an immediate consequence of part (a).

If we restrict to the special case of lattice systems, we recover a result of Daubechies [16, Equation 2.2.9].

Corollary 3. If $g \in L^{2}\left(\mathbf{R}^{d}\right)$ and $\alpha, \beta>0$ are such that $\mathcal{G}\left(g, \alpha \mathbf{Z}^{d} \times \beta \mathbf{Z}^{d}\right)$ is a frame for $L^{2}\left(\mathbf{R}^{d}\right)$, then

$$
A \leq \frac{\|g\|_{2}^{2}}{(\alpha \beta)^{d}} \leq B
$$

\subsection{Excess of Gabor Frames}

In this section we consider the excess of Gabor frames, and show that subsets with positive density may be removed from an overcomplete Gabor frame yet still leave a frame.

Theorem 6. Let $g \in L^{2}\left(\mathbf{R}^{d}\right)$ and $\Lambda \subset \mathbf{R}^{2 d}$ be such that $\mathcal{G}(g, \Lambda)$ is a Gabor frame for $L^{2}\left(\mathbf{R}^{d}\right)$. Then the following statements hold.

(a) If $D_{B}^{+}(\Lambda)>1$ then $\mathcal{G}(g, \Lambda)$ has infinite excess, and there exists an infinite subset $J \subset \Lambda$ such that $\mathcal{G}(g, \Lambda \backslash J)$ is a frame for $L^{2}\left(\mathbf{R}^{d}\right)$.

(b) If $g \in M^{1}$ and $D_{B}^{-}(\Lambda)>1$, then there exists $J \subset \Lambda$ with $D_{B}^{+}(J)=D_{B}^{-}(J)>0$ such that $\mathcal{G}(g, \Lambda \backslash J)$ is a frame for $L^{2}\left(\mathbf{R}^{d}\right)$.

\section{Proof.}

(a) By Theorem 3(c) we have $\mathcal{M}^{-}(\mathcal{G}(g, \Lambda))<1$. The result therefore follows from $[4$, Proposition 1].

(b) By Theorem 3(c) we have $\mathcal{M}^{+}(\mathcal{G}(g, \Lambda))<1$. Since $g \in M^{1}$, we have that $(\mathcal{G}(g, \Lambda), a)$ is $\ell^{1}$-self-localized by Theorem 2 . Hence the result follows from [4, Theorem 8].

\subsection{Localization and Structure of the Canonical Dual Frame}

In this section we study the structure of the canonical dual frame of an irregular Gabor frame.

First we introduce the notion of Gabor molecules. The term "molecule" arises from the convention that the generator $g$ of a Gabor system $\mathcal{G}(g, \Lambda)$ is often referred to as an "atom."

Definition 10. Let $\Lambda \subset \mathbf{R}^{2 d}$ and $f_{\lambda} \in L^{2}\left(\mathbf{R}^{d}\right)$ for $\lambda \in \Lambda$ be given. Then $\mathcal{F}=\left\{f_{\lambda}\right\}_{\lambda \in \Lambda}$ is a set of Gabor molecules if there exists an envelope function $\Gamma \in W\left(\mathcal{C}, \ell^{2}\right)$ such that

$$
\forall \lambda \in \Lambda, \quad \forall z \in \mathbf{R}^{2 d}, \quad\left|V_{\gamma} f_{\lambda}(z)\right| \leq \Gamma(z-\lambda) .
$$

Thus, if $\Gamma$ is concentrated around the origin in $\mathbf{R}^{2 d}$, then the STFT of $f_{\lambda}$ is concentrated around the point $\lambda$. Every Gabor system $\mathcal{G}(g, \Lambda)$ is a set of Gabor molecules, as $\left|V_{\gamma} g_{\lambda}(z)\right|=\left|V_{\gamma} g(z-\lambda)\right|$ for every $z, \lambda$. Sometimes the following equivalent definition is more convenient: $\mathcal{F}=\left\{M_{\omega} T_{x} f_{x \omega}\right\}_{(x, \omega) \in \Lambda}$ is a set of Gabor molecules if there exists $\Gamma \in W\left(\mathcal{C}, \ell^{2}\right)$ such that $\left|V_{\gamma} f_{x \omega}(z)\right| \leq \Gamma(z)$ for all $(x, \omega) \in \Lambda$ and $z \in \mathbf{R}^{2 d}$. 
The following lemma shows that the definition of Gabor molecules is unchanged if we replace the Gaussian window by any window function $\phi \in M^{1}$.

Lemma 3. Suppose $\mathcal{F}=\left\{f_{\lambda}\right\}_{\lambda \in \Lambda}$ is a set of Gabor molecules with envelope $\Gamma \in W\left(\mathcal{C}, \ell^{2}\right)$. If $\phi \in M^{1}$, then $\Gamma_{\phi}=\Gamma * V_{\phi} \gamma \in W\left(\mathcal{C}, \ell^{2}\right)$, and $\left|V_{\phi} f_{\lambda}(z)\right| \leq \Gamma_{\phi}(z-\lambda)$ for all $\lambda \in \Lambda$ and $z \in \mathbf{R}^{2 d}$.

Proof. Since $\gamma, \phi \in M^{1}$ we have $V_{\gamma} \gamma \in L^{1}\left(\mathbf{R}^{2 d}\right)$. Therefore $\Gamma_{\phi} \in W\left(\mathcal{C}, \ell^{2}\right) * L^{1} \subset W\left(\mathcal{C}, \ell^{2}\right)$ by [35, Theorem 11.1.5]. Further, by [35, Lemma 11.3.3] we have that $\left|V_{\phi} f_{\lambda}\right| \leq\left|V_{\gamma} f_{\lambda}\right| *\left|V_{\phi} \gamma\right|$, so

$$
\begin{aligned}
\left|V_{\phi} f_{\lambda}(z)\right| \leq\left(\left|V_{\gamma} f_{\lambda}\right| *\left|V_{\phi} \gamma\right|\right)(z) & =\int_{\mathbf{R}^{2 d}}\left|V_{\phi} f_{\lambda}(z-w)\right|\left|V_{\phi} \gamma(w)\right| d w \\
& \leq \int_{\mathbf{R}^{2 d}} \Gamma(z-w-\lambda)\left|V_{\phi} \gamma(w)\right| d w \\
& =\left(\Gamma *\left|V_{\phi} \gamma\right|\right)(z-\lambda)=\Gamma_{\phi}(z-\lambda) .
\end{aligned}
$$

Gröchenig and Leinert [40] proved that if $\Lambda$ is a lattice then the canonical dual frame of a lattice Gabor frame generated by a function $g \in M^{1}$ is generated by a dual window that also lies in $M^{1}$ (they also obtained weighted versions of this result). Their proof relied on deep results about symmetric Banach algebras. Here we extend this result to the more general setting of irregular Gabor frame sequences, and furthermore determine the structure of the dual frame (which in the lattice setting is simply another lattice Gabor frame). Note in particular that this result also applies to Gabor Riesz sequences.

Theorem 7. Let $g \in M^{1}$ and $\Lambda \subset \mathbf{R}^{2 d}$ be such that $\mathcal{G}(g, \Lambda)$ is a Gabor frame sequence in $L^{2}\left(\mathbf{R}^{d}\right)$, with canonical dual frame sequence $\tilde{\mathcal{G}}=\left\{\tilde{g}_{\lambda}\right\}_{\lambda \in \Lambda}$. Then the following statements hold:

(a) $\tilde{g}_{\lambda} \in M^{1}$ for all $\lambda \in \Lambda$,

(b) $\sup _{\lambda}\left\|\tilde{g}_{\lambda}\right\|_{M^{1}}<\infty$, and

(c) $\tilde{\mathcal{G}}$ is a set of Gabor molecules with respect to an envelope function $\Gamma \in W\left(\mathcal{C}, \ell^{1}\right)$.

Furthermore, the same conclusions hold when $\tilde{\mathcal{G}}$ is replaced by the canonical Parseval frame $S^{-1 / 2}(\mathcal{G}(g, \Lambda))$.

\section{Proof.}

(a) Since $g \in M^{1}$, we have by Theorem 2 that $(\mathcal{G}(g, \Lambda), a)$ is $\ell^{1}$-self-localized. Theorem 1 therefore implies that $(\tilde{\mathcal{G}}, a)$ is $\ell^{1}$-self-localized as well. Hence, by definition, there exists $r \in \ell^{1}(G)$ such that

$$
\left|\left\langle\tilde{g}_{\lambda}, \tilde{g}_{\mu}\right\rangle\right|<r_{a(\lambda)-a(\mu)} .
$$

Since $\mathcal{G}(g, \Lambda)$ is a Bessel sequence, $\Lambda$ has finite density by Theorem 1 . Thus $K=$ $\sup _{\lambda}\left|a^{-1}(\lambda)\right|<\infty$. For each $\lambda \in \Lambda$, the frame expansion of $\tilde{g}_{\lambda}$ is

$$
\tilde{g}_{\lambda}=\sum_{\mu \in \Lambda}\left\langle\tilde{g}_{\lambda}, \tilde{g}_{\mu}\right\rangle g_{\mu}
$$

with convergence in $L^{2}\left(\mathbf{R}^{d}\right)$. However, $\left\{\left\langle\tilde{g}_{\lambda}, \tilde{g}_{\mu}\right\rangle\right\}_{\mu \in \Lambda} \in \ell^{1}(\Lambda)$, so by [35, Theorem 12.1.8] the series on the right-hand side of (4.4) converges in $M^{1}$-norm, and therefore $\tilde{g}_{\lambda} \in M^{1}$. 
(b) Since translation and modulation are isometries in $M^{1}$-norm, we have by applying the triangle inequality to (4.4) that

$$
\begin{aligned}
\left\|\tilde{g}_{\lambda}\right\|_{M^{1}} & \leq \sum_{\mu \in \Lambda}\left|\left\langle\tilde{g}_{\lambda}, \tilde{g}_{\mu}\right\rangle\right|\left\|g_{\mu}\right\|_{M^{1}} \\
& =\sum_{j \in G} \sum_{\mu \in a^{-1}(j)}\left|\left\langle\tilde{g}_{\lambda}, \tilde{g}_{\mu}\right\rangle\right|\|g\|_{M^{1}} \leq K\|r\|_{\ell^{1}}\|g\|_{M^{1}}
\end{aligned}
$$

(c) Set

$$
\begin{aligned}
& \mathbf{Q}_{\alpha, \beta}(x, y)=[0, \alpha)^{d} \times[0, \beta)^{d}+(x, y), \\
& \mathbf{R}_{\alpha, \beta}(x, y)=[-\alpha, \alpha)^{d} \times[-\beta, \beta)^{d}+(x, y),
\end{aligned}
$$

and define

$$
\Gamma(z)=K \sum_{j \in G} r_{j} \sup _{w \in \mathbf{R}_{\alpha, \beta}(j)}\left|V_{\gamma} g(z-w)\right|, \quad z \in \mathbf{R}^{2 d} .
$$

Because $r \in \ell^{1}$ and $V_{\gamma} g \in W\left(\mathcal{C}, \ell^{1}\right)$, we have $\Gamma \in W\left(\mathcal{C}, \ell^{1}\right)$ as well.

Given $\mu \in \Lambda$, recall that $a(\mu)=\bar{\mu}$, so $\mu=a(\mu)+\tilde{\mu} \in \mathbf{Q}_{\alpha, \beta}(a(\mu))$. Also,

$$
\mathbf{Q}_{\alpha, \beta}(j+a(\lambda))=[0, \alpha)^{d} \times[0, \beta)^{d}+j+\lambda-\tilde{\lambda} \subset \mathbf{R}_{\alpha, \beta}(j+\lambda) .
$$

Therefore, taking the STFT of both sides of (4.4), we have

$$
\begin{aligned}
\left|V_{\gamma} \tilde{g}_{\lambda}(z)\right| & \leq \sum_{\mu \in \Lambda}\left|\left\langle\tilde{g}_{\lambda}, \tilde{g}_{\mu}\right\rangle\right|\left|V_{\gamma} g_{\mu}(z)\right| \\
& \leq \sum_{j \in G} \sum_{\mu \in a^{-1}(j)} r_{a(\mu)-a(\lambda)}\left|V_{\gamma} g(z-\mu)\right| \\
& \leq K \sum_{j \in G} r_{j-a(\lambda)} \sup _{w \in \mathbf{Q}_{\alpha, \beta}(j)}\left|V_{\gamma} g(z-w)\right| \\
& =K \sum_{j \in G} r_{j} \sup _{w \in \mathbf{Q}_{\alpha, \beta}(j+a(\lambda))}\left|V_{\gamma} g(z-w)\right| \\
& \leq K \sum_{j \in G} r_{j} \sup _{w \in \mathbf{R}_{\alpha, \beta}(j+\lambda)}\left|V_{\gamma} g(z-w)\right|=\Gamma(z-\lambda) .
\end{aligned}
$$

Thus $\tilde{\mathcal{G}}$ is a set of Gabor molecules.

Finally, by Theorem 1, the canonical Parseval frame $S^{-1 / 2}(\mathcal{G}(g, \Lambda))$ is $\ell^{1}$-self-localized, and computations similar to the ones above extend the results to the Parseval frame.

\subsection{Gabor Molecules}

We close our results on Gabor systems by noting that many of the results of the preceding sections proved for Gabor frames carry over to frames of Gabor molecules with only minor changes in proof.

Theorem 8. Let $\mathcal{F}=\left\{f_{\lambda}\right\}_{\lambda \in \Lambda}$ be a set of Gabor molecules with respect to an envelope $\Gamma \in W\left(\mathbf{C}, \ell^{2}\right)$. Let $\phi \in M^{1}$ and $\alpha, \beta>0$ be given. Then the following statements hold. 
(a) If $1 \leq p \leq 2$ and $\Gamma \in W\left(\mathcal{C}, \ell^{p}\right)$, then $(\mathcal{F}, a, \mathcal{G}(\phi, G))$ is $\ell^{p}$-localized. Further, $f_{\lambda} \in M^{p}$ for every $\lambda \in \Lambda$.

(b) If $\Gamma \in W\left(\mathcal{C}, \ell^{1}\right)$, then $(\mathcal{F}, a)$ is $\ell^{1}$-self-localized.

Theorem 9. Let $\mathcal{F}=\left\{f_{\lambda}\right\}_{\lambda \in \Lambda}$ be a set of Gabor molecules with respect to an envelope $\Gamma \in W\left(\mathcal{C}, \ell^{2}\right)$. If $\mathcal{F}$ is a frame for $L^{2}\left(\mathbf{R}^{d}\right)$ then the following statements hold.

(a) $1 \leq D_{B}^{-}(\Lambda) \leq D_{B}^{+}(\Lambda)<\infty$.

(b) $\mathcal{M}^{-}(\mathcal{F})=\frac{1}{D_{B}^{+}(\Lambda)}$ and $\mathcal{M}^{+}(\mathcal{F})=\frac{1}{D_{B}^{-}(\Lambda)}$.

(c) If $D_{B}^{+}(\Lambda)>1$ then $\mathcal{F}$ has infinite excess, and there exists an infinite subset $J \subset \Lambda$ such that $\left\{f_{\lambda}\right\}_{\lambda \in \Lambda \backslash J}$ is a frame for $L^{2}\left(\mathbf{R}^{d}\right)$.

(d) If $\Gamma \in W\left(\mathcal{C}, \ell^{1}\right)$ and $D_{B}^{-}(\Lambda)>1$, then there exists $J \subset \Lambda$ with $D_{B}^{+}(J)=D_{B}^{-}(J)>0$ such that $\left\{f_{\lambda}\right\}_{\lambda \in \Lambda \backslash J}$ is a frame for $L^{2}\left(\mathbf{R}^{d}\right)$.

(e) If $\Gamma \in W\left(\mathcal{C}, \ell^{1}\right)$ then the canonical dual frame $\tilde{\mathcal{F}}=\left\{\tilde{f}_{\lambda}\right\}_{\lambda \in \Lambda}$ is a set of Gabor molecules with respect to an envelope function $\tilde{\Gamma} \in W\left(\mathcal{C}, \ell^{1}\right)$.

\section{Relations Among the Localization and Approximation Properties}

We conclude this work by determining the relationships that hold among the localization and approximation properties described in Section 2. For the case that $\mathcal{F}$ and $\mathcal{E}$ are both frames for $H$ and the upper density $D^{+}(I, a)$ is finite, these relations can be summarized in the diagram in Figure 1.

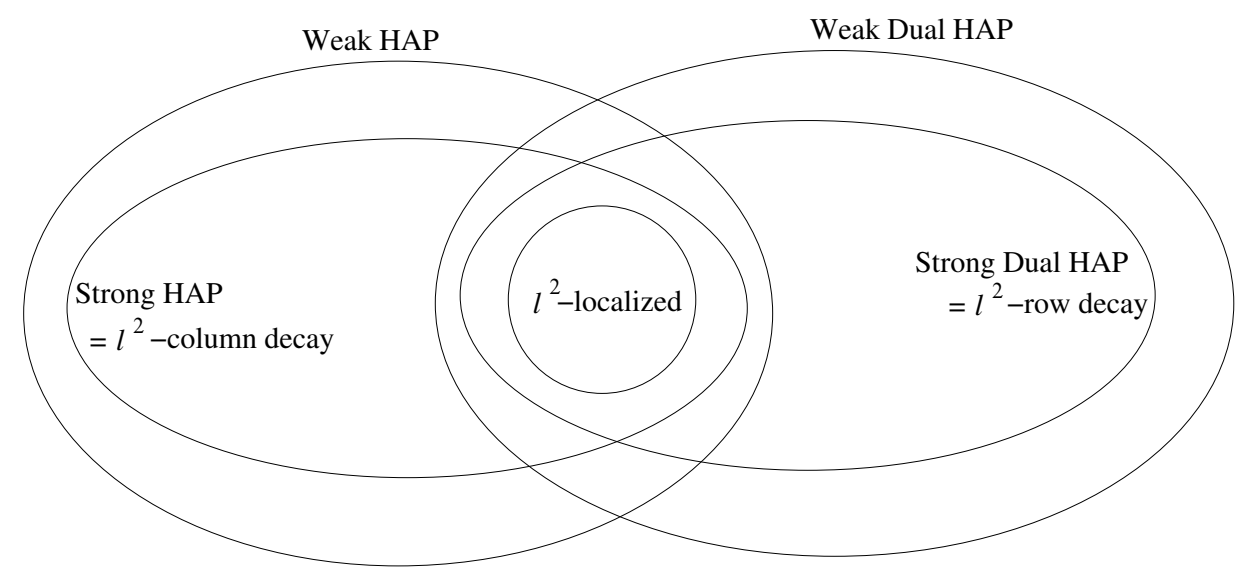

FIGURE 1 Relations among the localization and approximation properties for $p=2$, under the assumptions that $\mathcal{F}, \mathcal{E}$ are frames and $D^{+}(I, a)<\infty$. 


\subsection{Implications Among the Localization and Approximation Properties}

Theorem 10. Let $\mathcal{F}=\left\{f_{i}\right\}_{i \in I}$ and $\mathcal{E}=\left\{e_{j}\right\}_{j \in G}$ be sequences in $H$, and let $a: I \rightarrow G$ be an associated map. Then the following statements hold.

(a) If $\mathcal{F}$ is a frame for $H$, then $\ell^{2}$-column decay implies the strong HAP.

(b) If $\mathcal{F}$ is a frame for $H$ and $\sup _{j}\left\|e_{j}\right\|<\infty$, then the strong HAP implies $\ell^{2}$-column decay.

(c) If $\mathcal{E}$ is a frame for $H$, then $\ell^{2}$-row decay implies the strong dual HAP.

(d) If $\mathcal{E}$ is a frame for $H$ and $\sup _{i}\left\|f_{i}\right\|<\infty$, then the strong dual HAP implies $\ell^{2}$-row decay.

(e) If $\mathcal{F}$ is a frame for $H$, then the strong $H A P$ implies the weak HAP. If $\mathcal{F}$ is a Riesz basis for $H$, then the weak HAP implies the strong HAP.

(f) If $\mathcal{E}$ is a frame for $H$, then the strong dual HAP implies the weak dual HAP. If $\mathcal{E}$ is a Riesz basis for $H$, then the weak dual HAP implies the strong dual HAP.

(g) If $D^{+}(I, a)<\infty$ and $1 \leq p<\infty$, then $\ell^{p}$-localization implies both $\ell^{p}$-column and $\ell^{p}$-row decay.

Before giving the proof of Theorem 10, we note that in Section 5.2 we construct counterexamples to most of the converse implications of Theorem 10, including the following.

(a) There exist orthonormal bases $\mathcal{E}, \mathcal{F}$ such that $(\mathcal{F}, a, \mathcal{E})$ does not have $\ell^{2}$-column decay, and hence does not satisfy the strong HAP.

(b) There exists a frame $\mathcal{F}$ and orthonormal basis $\mathcal{E}$ such that $(\mathcal{F}, a, \mathcal{E})$ satisfies the weak HAP but not the strong HAP.

(c) There exists a frame $\mathcal{F}$ and orthonormal basis $\mathcal{E}$ such that $D^{+}(I, a)<\infty,(\mathcal{F}, a, \mathcal{E})$ has both $\ell^{2}$-column decay and $\ell^{2}$-row decay, but fails to have $\ell^{2}$-localization.

(d) There exists a Riesz basis $\mathcal{F}$ and orthonormal basis $\mathcal{E}$ such that $(\mathcal{F}, a, \mathcal{E})$ has $\ell^{2}$ column decay but not $\ell^{2}$-row decay.

\section{Proof of Theorem 10.}

(a) Assume $\mathcal{F}$ is a frame for $H$, with frame bounds $A, B$, and suppose that $(\mathcal{F}, a, \mathcal{E})$ has $\ell^{2}$-column decay. For each $j \in G$ we have $e_{j}=\sum_{i \in I}\left\langle e_{j}, f_{i}\right\rangle \tilde{f}_{i}$, so

$$
\begin{aligned}
\left\|e_{j}-\sum_{i \in I_{N_{\varepsilon}(j)}}\left\langle e_{j}, f_{i}\right\rangle \tilde{f}_{i}\right\|^{2} & =\left\|\sum_{i \in I \backslash I_{N_{\varepsilon}(j)}}\left\langle e_{j}, f_{i}\right\rangle \tilde{f}_{i}\right\|^{2} \\
& \leq \frac{1}{A} \sum_{i \in I \backslash I_{N_{\varepsilon}(j)}}\left|\left\langle e_{j}, f_{i}\right\rangle\right|^{2}<\frac{\varepsilon}{A} .
\end{aligned}
$$

Consequently, $\ell^{2}$-column decay implies the strong HAP.

(b) Assume that $\mathcal{F}$ is a frame for $H$, that $\mathcal{E}$ is uniformly bounded above in norm, and that $(\mathcal{F}, a, \mathcal{E})$ has the strong HAP. Let $S$ be the frame operator for $\mathcal{F}$. Since $S$ is bounded, $C=\sup _{j}\left\|S e_{j}\right\|<\infty$. Let $\tilde{\mathcal{F}}=\left\{\tilde{f}_{i}\right\}_{i \in I}$ be the canonical dual frame to $\mathcal{F}$. Since $\tilde{f}_{i}=S^{-1} f_{i}$ 
and $S$ is self-adjoint, we have

$$
\begin{aligned}
\sum_{i \in I \backslash I_{N_{\varepsilon}(j)}}\left|\left\langle e_{j}, f_{i}\right\rangle\right|^{2} & =\left\langle e_{j}, \sum_{i \in I \backslash I_{N_{\varepsilon}(j)}}\left\langle e_{j}, f_{i}\right\rangle f_{i}\right\rangle \\
& =\left\langle S e_{j}, \sum_{i \in I \backslash I_{N_{\varepsilon}(j)}}\left\langle e_{j}, f_{i}\right\rangle \tilde{f}_{i}\right\rangle \\
& \leq\left\|S e_{j}\right\|\left\|\sum_{i \in I \backslash I_{N_{\varepsilon}(j)}}\left\langle e_{j}, f_{i}\right\rangle \tilde{f}_{i}\right\| \\
& \leq C\left\|e_{j}-\sum_{i \in I_{N_{\varepsilon}(j)}}\left\langle e_{j}, f_{i}\right\rangle \tilde{f}_{i}\right\|<C \varepsilon
\end{aligned}
$$

Consequently, $(\mathcal{F}, a, \mathcal{E})$ has $\ell^{2}$-column decay.

(c), (d) These arguments are entirely symmetrical to the ones for (a), (b).

(e) Clearly the strong HAP trivially implies the weak HAP.

Suppose that $\mathcal{F}$ is a Riesz basis for $H$, and that $(\mathcal{F}, a, \mathcal{E})$ satisfies the weak HAP. Since $\tilde{\mathcal{F}}$ is also a Riesz basis for $H$, there exist constants $A^{\prime}, B^{\prime}$ such that

$$
A^{\prime} \sum_{i \in I}\left|a_{i}\right|^{2} \leq\left\|\sum_{i \in I} a_{i} \tilde{f}_{i}\right\|^{2} \leq B^{\prime} \sum_{i \in I}\left|a_{i}\right|^{2}
$$

for any square-summable sequence of scalars $\left(a_{i}\right)$. Fix any $\varepsilon>0$, and let $c_{i, j}$ be the numbers from (2.5). Then for any $j \in G$,

$$
\begin{aligned}
\varepsilon & >\left\|e_{j}-\sum_{i \in N_{\varepsilon}(j)} c_{i, j} \tilde{f}_{i}\right\|^{2} \\
& =\left\|e_{j}-\sum_{i \in N_{\varepsilon}(j)}\left\langle e_{j}, f_{i}\right\rangle \tilde{f}_{i}+\sum_{i \in N_{\varepsilon}(j)}\left(\left\langle e_{j}, f_{i}\right\rangle-c_{i, j}\right) \tilde{f}_{i}\right\|^{2} \\
& =\left\|\sum_{i \in I \backslash N_{\varepsilon}(j)}\left\langle e_{j}, f_{i}\right\rangle \tilde{f}_{i}+\sum_{i \in N_{\varepsilon}(j)}\left(\left\langle e_{j}, f_{i}\right\rangle-c_{i, j}\right) \tilde{f}_{i}\right\|^{2} \\
& \geq A^{\prime}\left(\sum_{i \in I \backslash N_{\varepsilon}(j)}\left|\left\langle e_{j}, f_{i}\right\rangle\right|^{2}+\sum_{i \in N_{\varepsilon}(j)}\left|\left\langle e_{j}, f_{i}\right\rangle-c_{i, j}\right|^{2}\right) \\
& \geq A^{\prime} \sum_{i \in I \backslash N_{\varepsilon}(j)}\left|\left\langle e_{j}, f_{i}\right\rangle\right|^{2} \\
& \geq \frac{A^{\prime}}{B^{\prime}}\left\|\sum_{i \in I \backslash N_{\varepsilon}(j)}\left\langle e_{j}, f_{i}\right\rangle \tilde{f}_{i}\right\|^{2} \\
& =\frac{A^{\prime}}{B^{\prime}}\left\|e_{j}-\sum_{i \in N_{\varepsilon}(j)}\left\langle e_{j}, f_{i}\right\rangle \tilde{f}_{i}\right\|^{2} .
\end{aligned}
$$

Hence $(\mathcal{F}, a, \mathcal{E})$ satisfies the strong HAP. 
(f) This argument is symmetrical to the one for (e).

(g) Assume that $(\mathcal{F}, a, \mathcal{E})$ is $\ell^{p}$-localized and that $D^{+}(I, a)<\infty$. Then we have $K=$ $\sup _{n \in G}\left|a^{-1}(n)\right|<\infty$. By definition of $\ell^{p}$-localization, there exists an $r \in \ell^{p}(G)$ such that $\left|\left\langle f_{i}, e_{j}\right\rangle\right| \leq r_{a(i)-j}$ for all $i \in I$ and $j \in G$. Given $\varepsilon>0$, let $N_{\varepsilon}$ be such that

$$
\sum_{\ell \in G \backslash S_{N_{\varepsilon}}(0)} r_{\ell}^{p}<\varepsilon
$$

Then

$$
\begin{aligned}
\sum_{i \in I \backslash I_{N_{\varepsilon}}(j)}\left|\left\langle f_{i}, e_{j}\right\rangle\right|^{p} & \leq \sum_{n \in G \backslash S_{N_{\varepsilon}}(j)} \sum_{i \in a^{-1}(n)} r_{a(i)-j}^{p} \\
& \leq K \sum_{n \in G \backslash S_{N_{\varepsilon}}(j)} r_{n-j}^{p} \\
& \leq K \varepsilon .
\end{aligned}
$$

Thus $(\mathcal{F}, a, \mathcal{E})$ has $\ell^{p}$-column decay. Additionally,

$$
\sum_{j \in G \backslash S_{N_{\varepsilon}}(a(i))}\left|\left\langle f_{i}, e_{j}\right\rangle\right|^{p} \leq \sum_{j \in G \backslash S_{N_{\varepsilon}}(a(i))} r_{a(i)-j}^{p} \leq \sum_{\ell \in G \backslash S_{N_{\varepsilon}}(0)} r_{\ell}^{p} \leq \varepsilon,
$$

so $(\mathcal{F}, a, \mathcal{E})$ has $\ell^{p}$-row decay as well.

\subsection{Counterexamples}

In this section, we provide examples showing that most of the implications of Theorem 10 are sharp, along with several other useful examples.

The following example constructs orthonormal bases $\mathcal{F}$ and $\mathcal{E}$ such that $(\mathcal{F}, a, \mathcal{E})$ does not satisfy the strong HAP.

Example 6. For each $n \in \mathbf{N}$, let $H_{n}$ be an $n$-dimensional Hilbert space with orthonormal basis $\left\{e_{j}^{n}\right\}_{j=1}^{n}$. Let $H=\ell^{2} \oplus \sum_{n=1}^{\infty} H_{n}$, the orthogonal direct sum of $\ell^{2}$ and the $H_{n}$. Let $\left\{e_{j}\right\}_{j \leq 0}$ be an orthonormal basis for $\ell^{2}$, and let $\left\{e_{j}\right\}_{j>0}$ be the orthonormal bases for the

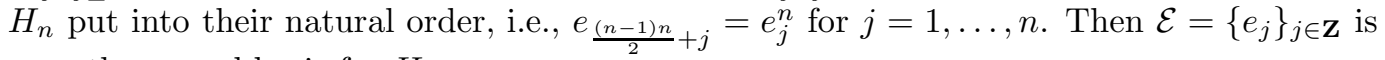
an orthonormal basis for $H$.

Let $\omega_{n}=e^{2 \pi i / n}$ be a primitive $n$th root of unity, and let $\left\{f_{k}^{n}\right\}_{k=1}^{n}$ be the harmonic orthonormal basis for $H_{n}$ given by

$$
f_{k}^{n}=\frac{1}{\sqrt{n}} \sum_{j=1}^{n} \omega_{n}^{j k} e_{j}^{n}, \quad k=1, \ldots, n .
$$

Let $\left\{f_{k}\right\}_{k>0}$ be the $f_{k}^{n}$ put in their natural order, and for $k \leq 0$ set $f_{k}=e_{k}$. Then $\mathcal{F}=\left\{f_{k}\right\}_{k \in \mathbf{Z}}$ is an orthonormal basis for $H$.

Let $a: \mathbf{Z} \rightarrow \mathbf{Z}$ be the identity map. Fix any $N>0$. If $j=\frac{(n-1) n}{2}+1$, then $\left\langle f_{k}, e_{j}\right\rangle=0$ for all $k<j$ or $k \geq j+n$. Since $I_{2 N}(j)=S_{2 N}(j)=[j-N, j+N) \cap \mathbf{Z}$, we therefore have 
for $n>N$ that

$$
\begin{aligned}
\sum_{k \in \mathbf{Z} \backslash I_{2 N}(j)}\left|\left\langle f_{k}, e_{j}\right\rangle\right|^{2} & =\sum_{k=j+N}^{j+n-1}\left|\left\langle f_{k}, e_{j}\right\rangle\right|^{2} \\
& =\sum_{k=N+1}^{n}\left|\left\langle f_{k}^{n}, e_{1}^{n}\right\rangle\right|^{2}=\sum_{k=N+1}^{n} \frac{1}{n}=\frac{n-N-1}{n} .
\end{aligned}
$$

This quantity approaches 1 as $n \rightarrow \infty$, so $(\mathcal{F}, a, \mathcal{E})$ fails to have $\ell^{2}$-column decay, and hence by Theorem 10 also fails the strong HAP.

The following example shows that the weak HAP need not imply the strong HAP if $\mathcal{F}$ is not a Riesz basis (compare to part (e) of Theorem 10). Note that in this example, $\mathcal{E}$ is actually an orthonormal basis for $H$.

Example 7. Let $\mathcal{E}$ and $\mathcal{F}$ be as in Example 6. Define

$$
g_{2 i}^{n}=\frac{1}{\sqrt{2}} e_{i}^{n}, \quad g_{2 i-1}^{n}=\frac{1}{\sqrt{2}} f_{i}^{n}, \quad i=1, \ldots, n .
$$

Let $\left\{g_{i}\right\}_{i>0}$ be the $g_{i}^{n}$ put in their natural order, and for $i \leq 0$ set $g_{i}=e_{i}$. Then $\mathcal{G}=\left\{g_{i}\right\}_{i \in \mathbf{Z}}$ is a Parseval frame for $H$, i.e., the frame bounds are $A=B=1$. In particular, $\mathcal{G}$ is its own dual frame.

Define $a: \mathbf{Z} \rightarrow \mathbf{Z}$ by $a(i)=i$ for $i \leq 0$ and

$$
a\left(\frac{(2 n-1) 2 n}{2}+2 i-1\right)=a\left(\frac{(2 n-1) 2 n}{2}+2 i\right)=\frac{(n-1) n}{2}+i, \quad i=1, \ldots, 2 n,
$$

i.e., $a$ associates the elements $g_{2 i-1}^{n}$ and $g_{2 i}^{n}$ of $\mathcal{G}$ with the element $e_{i}^{n}$ in $\mathcal{E}$.

Given any $j \in \mathbf{Z}$, we have $g_{j}=e_{j}$ for $j \leq 0$, and $\sqrt{2} g_{2 j}^{n}=e_{j}^{n}$ for $j>0$, so clearly $(\mathcal{G}, a, \mathcal{E})$ satisfies the weak HAP. However, given any $N>0$ we have

$$
\sum_{i \in \mathbf{Z} \backslash I_{2 N}(j)}\left|\left\langle g_{i}, e_{j}\right\rangle\right|^{2} \geq \frac{1}{2} \sum_{k \in \mathbf{Z} \backslash S_{2 N}(j)}\left|\left\langle f_{k}, e_{j}\right\rangle\right|^{2},
$$

and as we saw in Example 6, we cannot make this quantity arbitrarily small independently of $j$. Thus $(\mathcal{G}, a, \mathcal{E})$ fails the strong HAP.

The following example shows that the assumption of $\ell^{2}$-localization alone does not guarantee that the upper density is finite. In particular, this shows that the hypothesis in $\left[4\right.$, Theorem 4] that $\inf _{i}\left\|f_{i}\right\|>0$ is necessary.

Example 8. Let $\mathcal{E}=\left\{e_{n}\right\}_{n \in \mathbf{Z}}$ be an orthonormal basis for $H$ and let $\mathcal{F}=\left\{e_{n}\right\}_{n>0} \cup$ $\left\{2^{n} e_{0}\right\}_{n \leq 0}$. Note that $\mathcal{F}$ is a frame sequence in $H$. If we let $a(i)=i$ for $i>0$ and $a(i)=0$ for $i \leq 0$, then $\left|a^{-1}(0)\right|=\infty$, so $D^{+}(I, a)=\infty$. On the other hand, $\sup _{i \in \mathbf{Z}}\left|\left\langle f_{i}, e_{j+a(i)}\right\rangle\right|=1$ if $j=0$ and 0 otherwise, so $(\mathcal{F}, a, \mathcal{E})$ is $\ell^{2}$-localized.

The following example shows that the converse of part $(\mathrm{g})$ of Theorem 10 fails in general, i.e., $\ell^{2}$-column decay combined with $\ell^{2}$-row decay does not imply $\ell^{2}$-localization, even if $D^{+}(I, a)<\infty$.

Example 9. Let $\mathcal{E}=\left\{e_{j}\right\}_{j \in \mathbf{Z}}$ be an orthonormal basis for $H$, and define $\mathcal{F}=\left\{f_{j}\right\}_{j \in \mathbf{Z}}$ by

$$
f_{j}=e_{j}+\left(\frac{1}{4+|j|}\right)^{1 / 2} e_{-j}, \quad j \in \mathbf{Z}
$$


Let $a: \mathbf{Z} \rightarrow \mathbf{Z}$ be the identity map. Then $D^{+}(a, \mathbf{Z})=1$, and $I_{N}(j)=S_{N}(j)$ for all $j$ and $N$. For $j \neq 0$, we have

$$
\sup _{i \in \mathbf{Z}}\left|\left\langle f_{i}, e_{2 j+i}\right\rangle\right|^{2}=\left|\left\langle f_{-j}, e_{j}\right\rangle\right|^{2}=\frac{1}{4+|j|},
$$

so $(\mathcal{F}, a, \mathcal{E})$ is not $\ell^{2}$-localized. On the other hand, since $\left\langle f_{i}, e_{j}\right\rangle \neq 0$ only when $i= \pm j$, we have

$$
\sum_{i \in \mathbf{Z} \backslash S_{N_{\varepsilon}}(j)}\left|\left\langle f_{i}, e_{j}\right\rangle\right|^{2}= \begin{cases}0, & -\frac{N}{4}<j \leq \frac{N}{4}, \\ \frac{1}{4+|j|}, & \text { otherwise. }\end{cases}
$$

By taking $N_{\varepsilon}$ large enough, we can make this quantity arbitrarily small, independently of $j$. Thus $(\mathcal{F}, a, \mathcal{E})$ has $\ell^{2}$-column decay, and a similar argument shows it has $\ell^{2}$-row decay.

Note that no other choice for the map $a$ would help in this example, for if $(\mathcal{F}, a, \mathcal{E})$ has $\ell^{2}$-column decay, then $\sup _{j}|a(j)-j|<\infty$. Thus $a$ can only be a bounded perturbation of the identity. For such an $a$, there always exists an $N$ sufficiently large so that for $|j|>N$ an inequality similar to (5.1) will hold.

Note also in this example that $\mathcal{F}$ is a frame. For, given $f \in H$ we have

$$
\sum_{j \in \mathbf{Z}}\left|\left\langle f, f_{j}-e_{j}\right\rangle\right|^{2}=\sum_{j \in \mathbf{Z}}\left(\frac{1}{4+|j|}\right)\left|\left\langle f, e_{-j}\right\rangle\right|^{2} \leq \frac{1}{4} \sum_{j \in \mathbf{Z}}\left|\left\langle f, e_{j}\right\rangle\right|^{2}=\frac{1}{4}\|f\|^{2},
$$

and therefore, by the triangle inequality,

$$
\begin{aligned}
\left(\sum_{j \in \mathbf{Z}}\left|\left\langle f, f_{j}\right\rangle\right|^{2}\right)^{1 / 2} & \geq\left(\sum_{j \in \mathbf{Z}}\left|\left\langle f, e_{j}\right\rangle\right|^{2}\right)^{1 / 2}-\left(\sum_{j \in \mathbf{Z}}\left|\left\langle f, f_{j}-e_{j}\right\rangle\right|^{2}\right)^{1 / 2} \\
& \geq\|f\|-\frac{1}{2}\|f\|=\frac{1}{2}\|f\| .
\end{aligned}
$$

Thus $\mathcal{F}$ has a lower frame bound of $1 / 4$, and a similar calculation shows it has an upper frame bound of $9 / 4$.

The following example shows that $\ell^{2}$-column decay does not imply $\ell^{2}$-row decay. By interchanging the roles of $\mathcal{F}$ and $\mathcal{E}$ in this example, we also see that $\ell^{2}$-row decay does not imply $\ell^{2}$-column decay.

Example 10. Choose an orthonormal basis for $H$ and index it as

$$
\mathcal{E}=\operatorname{span}\left\{e_{j}^{n}\right\}_{n \in \mathbf{N}, j=1, \ldots, n}
$$

Set $H_{n}=\operatorname{span}\left\{e_{j}^{n}\right\}_{j=1, \ldots, n}$. Define

$$
f_{i}^{n}= \begin{cases}e_{1}^{n}, & i=1, \\ \frac{1}{2 \sqrt{n}} e_{1}^{n}+e_{i}^{n}, & i=2, \ldots, n,\end{cases}
$$

and

$$
\tilde{f}_{i}^{n}= \begin{cases}e_{1}^{n}-\frac{1}{2 \sqrt{n}} \sum_{j=2}^{n} e_{j}^{n}, & i=1, \\ e_{i}^{n}, & i=2, \ldots, n .\end{cases}
$$


Clearly $f_{i}^{n}, \tilde{f}_{i}^{n} \in H_{n}$, and a straightforward calculation shows that $\left\{f_{i}^{n}\right\}_{i=1}^{n}$ and $\left\{\tilde{f}_{i}^{n}\right\}_{i=1}^{n}$ are biorthogonal sequences in $H_{n}$. Since $H_{n}$ is $n$-dimensional, this shows that these are dual Riesz bases for $H_{n}$. Given any scalars $\left\{a_{i}\right\}_{i=1}^{n}$, we have

$$
\begin{aligned}
\left\|\sum_{i=1}^{n} a_{i} f_{i}^{n}\right\| & \leq\left\|\frac{\sum_{i=1}^{n} a_{i}}{2 \sqrt{n}} e_{1}^{n}\right\|+\left\|\sum_{i=1}^{n} a_{i} e_{i}^{n}\right\| \\
& \leq \frac{\sum_{i=1}^{n}\left|a_{i}\right|}{2 \sqrt{n}}+\left(\sum_{i=1}^{n}\left|a_{i}\right|^{2}\right)^{1 / 2} \leq \frac{3}{2}\left(\sum_{i=1}^{n}\left|a_{i}\right|^{2}\right)^{1 / 2},
\end{aligned}
$$

and similarly $\left\|\sum_{i=1}^{n} a_{i} f_{i}^{n}\right\| \geq \frac{1}{2}\left(\sum_{i=1}^{n}\left|a_{i}\right|^{2}\right)^{1 / 2}$. Thus $\left\{f_{i}^{n}\right\}_{i=1}^{n}$ has Riesz bounds $\frac{1}{2}, \frac{3}{2}$. Since $H$ is the orthogonal direct sum of the $H_{n}$ and the Riesz bounds are independent of $n$, we conclude that $\mathcal{F}=\left\{f_{i}^{n}\right\}_{n \in \mathbf{N}, i=1, \ldots, n}$ and $\tilde{\mathcal{F}}=\left\{\tilde{f}_{i}^{n}\right\}_{n \in \mathbf{N}, i=1, \ldots, n}$ are dual Riesz bases for $H$.

Another direct calculation shows that

$$
\left|\left\langle f_{i}^{m}, e_{j}^{n}\right\rangle\right|= \begin{cases}1, & i=j, m=n \\ \frac{1}{2 \sqrt{n}}, & m=n, j=1, i=2, \ldots, n, \\ 0, & \text { otherwise. }\end{cases}
$$

Consequently, given any $N$, we have for each $n>N$ and $j=1, \ldots, n$ that

$$
\sum_{m>N} \sum_{i=1}^{m}\left|\left\langle f_{i}^{m}, e_{j}^{n}\right\rangle\right|^{2}=\left.\sum_{i=1}^{n}\left\langle f_{i}^{n}, e_{j}^{n}\right\rangle\right|^{2}=\frac{1}{4 n}
$$

while for $n \leq N$ this sum is zero. Hence, by taking $N$ large enough this sum is less than $\varepsilon$ independently of $n \in \mathbf{N}$ and $j=1, \ldots, n$. Thus, with $a$ as the identity map, $(\mathcal{F}, a, \mathcal{E})$ has $\ell^{2}$-row decay. On the other hand, if $m>N$ then we have for each $i=1, \ldots, n$ that

$$
\sum_{n>N} \sum_{j=1}^{n}\left|\left\langle f_{i}^{m}, e_{j}^{n}\right\rangle\right|^{2}=\sum_{j=1}^{n}\left|\left\langle f_{i}^{n}, e_{j}^{n}\right\rangle\right|^{2}=\sum_{j=2}^{n} \frac{1}{4 n}=\frac{n-1}{4 n} .
$$

After mapping the index set of $\mathcal{E}$ and $\mathcal{F}$ onto $\mathbf{Z}$, similarly to Example 6, we conclude that $(\mathcal{F}, a, \mathcal{E})$ does not have $\ell^{2}$-row decay.

The following example illustrates the importance of the map $a$ in determining localization properties.

Example 11. Let $\mathcal{F}=\left\{f_{n}\right\}_{n \in \mathbf{Z}}$ be an orthonormal basis for $H$, and define $a: \mathbf{Z} \rightarrow \mathbf{Z}$ by $a(2 n)=a(2 n+1)=n$. Then $(\mathcal{F}, a)$ is $\ell^{1}$-self-localized, and by Example 4 we have $\mathcal{M}^{ \pm}(\mathcal{F})=1$. However, $D^{ \pm}(I, a)=2$. Hence, by [4, Theorem 6(c)], there cannot be any Riesz basis $\mathcal{E}$ such that $(\mathcal{F}, a, \mathcal{E})$ has both $\ell^{2}$-column decay and $\ell^{2}$-row decay. In particular $(\mathcal{F}, a, \mathcal{E})$ cannot be $\ell^{2}$-localized for any $\operatorname{Riesz}$ basis $\mathcal{E}$, and $(\mathcal{F}, a, \mathcal{F})$ is not $\ell^{2}$-localized.

However, if we let $\mathcal{E}=\left\{f_{2 n}\right\}_{n \in \mathbf{Z}}$, then $\mathcal{E}$ is a Riesz sequence (but not a Riesz basis), and $(\mathcal{F}, a, \mathcal{E})$ is $\ell^{1}$-localized. Since $\mathcal{E}$ is a Riesz sequence and $\overline{\operatorname{span}}(\mathcal{F})=H$, we have $\mathcal{M}^{ \pm}(\mathcal{E})=\mathcal{M}(\mathcal{E} ; p, c)=1$ by Example $4(\mathrm{~b})$. On the other hand, since $P_{\mathcal{E}}\left(f_{2 n}\right)=1$ and $P_{\mathcal{E}}\left(f_{2 n+1}\right)=0$, it follows directly that $\mathcal{M}_{\mathcal{E}}(\mathcal{F} ; p, c)=\frac{1}{2}$. Thus $\mathcal{M}_{\mathcal{E}}(\mathcal{F} ; p, c) D(p, c)=1$, in accordance with [4, Theorem 5].

The following example shows that $\ell^{1}$-localization with respect to the canonical dual frame does not imply $\ell^{1}$-self-localization. 
Example 12. Let $\mathcal{E}=\left\{e_{i}\right\}_{i \in \mathbf{Z}}$ be an orthonormal basis for $H$, and let $a: \mathbf{Z} \rightarrow \mathbf{Z}$ be the identity map. Fix $\frac{1}{2}<c_{0}<1$, and for $i \neq 0$ choose $c_{i}>0$ in such a way that

$$
\sum_{i \in \mathbf{Z}} c_{i}^{2}=1 \quad \text { and } \quad \sum_{i \in \mathbf{Z}} c_{i}=\infty .
$$

Define

$$
f_{0}=\sum_{i \in \mathbf{Z}} c_{i} e_{i} \quad \text { and } \quad f_{i}=e_{i} \text { for } i \neq 0 .
$$

If we set $T\left(e_{i}\right)=f_{i}$, then $T$ extends to a bounded mapping on $H$. Further, if $f=$ $\sum_{i}\left\langle f, e_{i}\right\rangle e_{i} \in H$, then

$$
\begin{aligned}
\|(\mathbf{1}-T) f\|^{2} & =\left\|\left\langle f, e_{0}\right\rangle\left(e_{0}-f_{0}\right)\right\|^{2} \\
& =\left|\left\langle f, e_{0}\right\rangle\right|^{2}\left(\left|1-c_{0}\right|^{2}+\sum_{i \neq 0} c_{i}^{2}\right) \leq\left(2-2 c_{0}\right)\|f\|^{2},
\end{aligned}
$$

so $\|\mathbf{1}-T\| \leq 2-2 c_{0}<1$. Hence $T$ is a continuous bijection of $H$ onto itself, so $\mathcal{F}=\left\{f_{i}\right\}_{i \in \mathbf{Z}}$ is a Riesz basis for $H$. Therefore $\left\langle f_{i}, \tilde{f}_{j}\right\rangle=\delta_{i j}$, so $(\mathcal{F}, a)$ is $\ell^{1}$-localized with respect to its dual frame. However, $\left\langle f_{0}, f_{j}\right\rangle=c_{j}$, so $(\mathcal{F}, a)$ is not $\ell^{1}$-self-localized.

\section{Appendix}

\section{A. Ultrafilters}

In this appendix we provide a brief review of ultrafilters and their basic properties. For additional information, we refer to [46, Chapter 3]. Filters were introduced by H. Cartan [9, $10]$ in order to characterize continuous functions on general topological spaces. Soon after, it was realized that the set of ultrafilters endowed with the proper topology is the Stone-Cech compactification of a discrete (or more generally, a completely regular) topological space. In the following we will restrict our attention to ultrafilters over the natural numbers $\mathbf{N}$.

Definition A.1. A collection $p$ of subsets of $\mathbf{N}$ is a filter if:

(a) $\emptyset \notin p$,

(b) if $A, B \in p$ then $A \cap B \in p$,

(c) if $A \in p$ and $A \subset B \subset \mathbf{N}$, then $B \in p$.

A filter $p$ is an ultrafilter if it is maximal in the sense that:

(d) if $p^{\prime}$ is a filter on $\mathbf{N}$ such that $p \subset p^{\prime}$, then $p^{\prime}=p$,

or, equivalently, if

(d') for any $A \subset \mathbf{N}$, either $A \in p$ or $\mathbf{N} \backslash A \in p$ (but not both, because of properties a and $\mathrm{b})$.

The set of ultrafilters is denoted by $\beta \mathbf{N}$. 
Definition A.2. Given any $n \in \mathbf{N}, e_{n}=\{A \subset \mathbf{N}: n \in A\}$ is an ultrafilter, called a principal ultrafilter. It is straightforward to show that any ultrafilter $p$ that contains a finite set must be one of these principal ultrafilters. An ultrafilter which contains no finite sets is called free. The set of free ultrafilters is denoted by $\mathbf{N}^{*}$.

Our main use for ultrafilters is that they provide a notion of convergence for arbitrary sequences.

Definition A.3. Let $p \in \beta \mathbf{N}$ be an ultrafilter. Then we say that a sequence $\left\{c_{k}\right\}_{k \in \mathbf{N}}$ of complex numbers converges to $c \in \mathbf{C}$ with respect to $p$ if for every $\varepsilon>0$ there exists a set $A \in p$ such that $\left|c_{k}-c\right|<\varepsilon$ for all $k \in A$. In this case we write $c=p$ - $\lim _{k \in \mathbf{N}} c_{k}$ or simply $c=p-\lim c_{k}$.

The following proposition summarizes the basic properties of convergence with respect to an ultrafilter.

Proposition A.1. Let $p \in \beta \mathbf{N}$ be an ultrafilter. Then the following statements hold.

(a) Every bounded sequence of complex scalars $\left\{c_{k}\right\}_{k \in \mathbf{N}}$ converges with respect to $p$ to some $c \in \mathbf{C}$.

(b) p-limits are unique.

(c) If $p=e_{n}$ is a principal ultrafilter, then $p$ - $\lim c_{k}=c_{n}$.

(d) If $\left\{c_{k}\right\}_{k \in \mathbf{N}}$ is a convergent sequence in the usual sense, $p$ is a free ultrafilter, and $\lim _{k \rightarrow \infty} c_{k}=c$, then $p$-lim $c_{k}=c$.

(e) If $\left\{c_{k}\right\}_{k \in \mathbf{N}}$ is a bounded sequence and $p$ is a free ultrafilter, then $p$ - $\lim _{k \in \mathbf{N}} c_{k}$ is an accumulation point of $\left\{c_{k}\right\}_{k \in \mathbf{N}}$.

(f) If $c$ is an accumulation point of a bounded sequence $\left\{c_{k}\right\}_{k \in \mathbf{N}}$, then there exists a free ultrafilter $p$ such that $p$ - $\lim c_{k}=c$. In particular, there exists an ultrafilter $p$ such that $p$ - $\lim c_{k}=\lim \sup c_{k}$, and there exists an ultrafilter $q$ such that $q$-lim $c_{k}=$ $\liminf c_{k}$.

(g) $\quad p$-limits are linear, i.e., $p$ - $\lim \left(a c_{k}+b d_{k}\right)=a p$ - $\lim c_{k}+b p$-lim $d_{k}$.

(h) $p$-limits respect products, i.e., $p$ - $\lim \left(c_{k} d_{k}\right)=\left(p-\lim c_{k}\right)\left(p\right.$-lim $\left.d_{k}\right)$.

\section{Acknowledgments}

We gratefully acknowledge conversations with Karlheinz Gröchenig and Massimo Fornasier on localization of frames, and thank them for providing us with preprints of their articles. We thank Hans Feichtinger, Norbert Kaiblinger, Gitta Kutyniok, and Henry Landau for conversations regarding the details of our arguments, and also acknowledge helpful conversations with Akram Aldroubi, Carlos Cabrelli, Mark Lammers, Ursula Molter, and Kasso Okoudjou.

\section{References}

[1] L. Baggett (1990). Processing a radar signal and representations of the discrete Heisenberg group, Colloq. Math. 60/61, 195-203. 
[2] R. Balan, P. G. Casazza, C. Heil, and Z. Landau (2003). Deficits and excesses of frames, Adv. Comput. Math. 18, 93-116.

[3] R. Balan, P. G. Casazza, C. Heil, and Z. Landau (2003). Excesses of Gabor frames, Appl. Comput. Harmon. Anal. 14, 87-106.

[4] R. Balan, P. G. Casazza, C. Heil, and Z. Landau (2006). Density, overcompleteness, and localization of frames, I. Theory, J. Fourier Anal. Appl. 12, 105-143.

[5] R. Balan, P. G. Casazza, C. Heil, and Z. Landau (2006). Density, overcompleteness, and localization of frames, Electron. Res. Announc. Amer. Math. Soc., to appear.

[6] J. J. Benedetto, W. Czaja, and A. Ya. Maltsev (2003), The Balian-Low theorem for the symplectic form on $\mathbf{R}^{2 d}$, J. Math. Physics 44, 1735-1750.

[7] J. J. Benedetto, C. Heil, and D. F. Walnut (1995). Differentiation and the Balian-Low theorem, J. Fourier Anal. Appl. 1, 355-402.

[8] M. Bownik and Z. Rzeszotnik (2003). The spectral function of shift-invariant spaces, Michigan Math. J. 51, 387-414.

[9] H. Cartan (1937). Théorie des filtres, C. R. Acad. Sci. Paris 205, 595-598.

[10] H. Cartan (1937). Filtres et ultrafiltres, C. R. Acad. Sci. Paris 205, 777-779.

[11] P. G. Casazza (2000). The art of frame theory, Taiwanese J. Math. 4, 129-201.

[12] P. G. Casazza and N. J. Kalton (2002). Roots of complex polynomials and Weyl-Heisenberg frame sets, Proc. Amer. Math. Soc. 130, 2313-2318.

[13] O. Christensen (2003). An Introduction to Frames and Riesz Bases, Birkhäuser, Boston.

[14] O. Christensen, B. Deng, and C. Heil (1999). Density of Gabor frames, Appl. Comput. Harmon. Anal. 7, 292-304.

[15] O. Christensen, S. Favier, and Z. Felipe (2001). Irregular wavelet frames and Gabor frames, Approx. Theory Appl. (N.S.) 17, 90-101.

[16] I. Daubechies (1990). The wavelet transform, time-frequency localization and signal analysis, IEEE Trans. Inform. Theory 39, 961-1005.

[17] I. Daubechies (1992). Ten Lectures on Wavelets, SIAM, Philadelphia.

[18] I. Daubechies, A. Grossmann, and Y. Meyer (1986). Painless nonorthogonal expansions, J. Math. Phys. 27, 1271-1283.

[19] I. Daubechies, H. Landau, and Z. Landau (1995). Gabor time-frequency lattices and the Wexler-Raz identity, J. Fourier Anal. Appl. 1, 437-478.

[20] B. Deng and C. Heil (2000). Density of Gabor Schauder bases, in Wavelet Applications in Signal and Image Processing VIII (San Diego, CA, 2000), A. Aldroubi at al., Eds., Proc. SPIE 4119, SPIE, Bellingham, WA, 153-164.

[21] H. G. Feichtinger (1980). Banach convolution algebras of Wiener type, in Functions, Series, Operators, Proc. Conf. Budapest 38, Colloq. Math. Soc. János Bolyai, 509-524.

[22] H. G. Feichtinger (1981). On a new Segal algebra, Monatsh. Math. 92, 269-289.

[23] H. G. Feichtinger (1987). Banach spaces of distributions defined by decomposition methods, II, Math. Nachr. 132, 207-237.

[24] H. G. Feichtinger (1989). Atomic characterizations of modulation spaces through Gabor-type representations, Rocky Mountain J. Math. 19, 113-125.

[25] H. G. Feichtinger (1990). Generalized amalgams, with applications to Fourier transform, Canad. J. Math. 42, 395-409.

[26] H. G. Feichtinger (1992). Wiener amalgams over Euclidean spaces and some of their applications, in Function spaces (Edwardsville, IL, 1990), Lecture Notes in Pure and Appl. Math. 136, Dekker, New York, 123-137.

[27] H. G. Feichtinger (2003). Modulation spaces on locally compact Abelian groups, in Wavelets and their Applications (Chennai, January 2002), M. Krishna, R. Radha and S. Thangavelu, Eds., Allied Publishers, New Delhi, 1-56.

[28] H. G. Feichtinger and P. Gröbner (1985). Banach spaces of distributions defined by decomposition methods, I, Math. Nachr. 123, 97-120.

[29] H. G. Feichtinger and K. Gröchenig (1989). Banach spaces related to integrable group representations and their atomic decompositions, I, J. Funct. Anal. 86, 307-340. 
[30] H. G. Feichtinger and K. Gröchenig (1989). Banach spaces related to integrable group representations and their atomic decompositions, II, Monatsh. Math. 108, 129-148.

[31] H. G. Feichtinger and K. Gröchenig (1997). Gabor frames and time-frequency analysis of distributions, J. Funct. Anal. 146, 464-495.

[32] H. G. Feichtinger and G. Zimmermann (1998). A Banach space of test functions for Gabor analysis, in Gabor Analysis and Algorithms: Theory and Applications, H. G. Feichtinger and T. Strohmer, Eds., Birkhäuser, Boston, 123-170.

[33] M. Fornasier (2003). Constructive methods for numerical applications in signal processing and homogenization problems, Ph.D. Thesis, U. Padua.

[34] K. Gröchenig (1993). Irregular sampling of wavelet and short-time Fourier transforms, Constr. Approx. 9, 283-297.

[35] K. Gröchenig (2001). Foundations of Time-Frequency Analysis, Birkhäuser, Boston.

[36] K. Gröchenig (2003). Localized frames are finite unions of Riesz sequences, Adv. Comput. Math. 18, 149-157.

[37] K. Gröchenig (2004). Localization of frames, Banach frames, and the invertibility of the frame operator, J. Fourier Anal. Appl. 10, 105-132.

[38] K. Gröchenig and M. Fornasier (2005). Intrinsic localization of frames, Constr. Approx. 22, 395-415.

[39] K. Gröchenig, D. Han, C. Heil, and G. Kutyniok (2002). The Balian-Low Theorem for symplectic lattices in higher dimensions, Appl. Comput. Harmon. Anal., 13, 169-176.

[40] K. Gröchenig and M. Leinert (2004). Wiener's lemma for twisted convolution and Gabor frames, $J$. Amer. Math. Soc. 17, 1-18.

[41] K. Gröchenig and H. Razafinjatovo (1996). On Landau's necessary density conditions for sampling and interpolation of band-limited functions, J. London Math. Soc. (2) 54, 557-565.

[42] D. Han and Y. Wang (2001). Lattice tiling and the Weyl-Heisenberg frames, Geom. Funct. Anal. 11, $742-758$.

[43] C. Heil (2003). An introduction to weighted Wiener amalgams, in Wavelets and their Applications (Chennai, January 2002), M. Krishna, R. Radha and S. Thangavelu, Eds., Allied Publishers, New Delhi, 183-216.

[44] C. Heil (2006). On the history of the Density Theorem for Gabor frames, preprint.

[45] C. E. Heil and D. F. Walnut (1989). Continuous and discrete wavelet transforms, SIAM Review 31, 628-666.

[46] N. Hindman and D. Strauss (1998). Algebra in the Stone-Čech Compactification, de Gruyter Expositions in Mathematics 27, Walter de Gruyter and Co., Berlin.

[47] A. J. E. M. Janssen (1994). Signal analytic proofs of two basic results on lattice expansions, Appl. Comput. Harmon. Anal. 1, 350-354.

[48] A. J. E. M. Janssen (1995). Duality and biorthogonality for Weyl-Heisenberg frames, J. Fourier Anal. Appl. 1, 403-436.

[49] A. J. E. M. Janssen (1998). A density theorem for time-continuous filter banks, in Signal and image representation in combined spaces, Y. Y. Zeevi and R. R. Coifman, Eds., Wavelet Anal. Appl. 7, Academic Press, San Diego, CA, 513-523.

[50] A. J. E. M. Janssen (2003). On generating tight Gabor frames at critical density, J. Fourier Anal. Appl. 9, 175-214.

[51] A. J. E. M. Janssen and T. Strohmer (2002). Hyperbolic secants yield Gabor frames, Appl. Comput. Harmon. Anal. 12, 259-267.

[52] H. Landau (1993). On the density of phase-space expansions, IEEE Trans. Inform. Theory 39, 11521156.

[53] Y. Liu and Y. Wang (2003). The uniformity of non-uniform Gabor bases, Adv. Comput. Math. 18, 345-355.

[54] Yu. I. Lyubarskii (1992). Frames in the Bargmann space of entire functions, in Entire and subharmonic functions, Amer. Math. Soc., Providence, RI, 167-180.

[55] J. Ramanathan and T. Steger (1995). Incompleteness of sparse coherent states, Appl. Comput. Harmon. Anal. 2, 148-153.

[56] M. Rieffel (1981). Von Neumann algebras associated with pairs of lattices in Lie groups, Math. Ann. 
257, 403-418.

[57] A. Ron and Z. Shen (1997). Weyl-Heisenberg frames and Riesz bases in $L^{2}\left(\mathbf{R}^{d}\right)$, Duke Math. J. 89, 237-282.

[58] K. Seip (1992). Density theorems for sampling and interpolation in the Bargmann-Fock space I, $J$. Reine Angew. Math. 429, 91-106.

[59] K. Seip and R. Wallstén (1992). Sampling and interpolation in the Bargmann-Fock space II, J. Reine Angew. Math. 429, 107-113.

[60] W. Sun and X. Zhou (2002). Irregular wavelet/Gabor frames, Appl. Comput. Harmon. Anal. 13, 63-76.

[61] W. Sun and X. Zhou(2003). Irregular Gabor frames and their stability, Proc. Amer. Math. Soc. 131, 2883-2893.

[62] R. Young (2001). An Introduction to Nonharmonic Fourier Series, Revised 1st Edition, Academic Press, San Diego

Received June 14, 2005

Siemens Corporate Research, 755 College Road East, Princeton, NJ 08540 e-mail: radu.balan@siemens.com

Department of Mathematics, University of Missouri, Columbia, MO 65211 e-mail: pete@math.missouri.edu

School of Mathematics, Georgia Institute of Technology, Atlanta, GA 30332 e-mail: heil@math.gatech.edu

Department of Mathematics R8133, The City College of New York, Convent Ave at 138th Street, New York, NY 10031 e-mail: landau@sci.ccny.cuny.edu 